\title{
Korringa-Kohn-Rostoker Green-function formalism for ballistic transport
}

\author{
Phivos Mavropoulos, ${ }^{1}$ Nikolaos Papanikolaou, ${ }^{2}$ and Peter H. Dederichs ${ }^{1}$ \\ ${ }^{1}$ Institut für Festkörperforschung, Forschungszentrum Jülich, D-52425 Jülich, Germany \\ ${ }^{2}$ Institute of Microelectronics, NCSR “Demokritos," GR-15310 Ag. Paraskevi, Attiki, Greece
}

(Received 26 June 2003; revised manuscript received 26 November 2003; published 17 March 2004)

\begin{abstract}
We develop a method for the calculation of ballistic transport from first principles. The multiple scattering screened Korringa-Kohn-Rostoker (KKR) method is combined with a Green-function formulation of the Landauer approach for the ballistic transport. We obtain an efficient $\mathrm{O}(N)$ algorithm for the calculation of ballistic conductance through a scattering region connected to semi-infinite crystalline leads. In particular we generalize the results of Baranger and Stone in the case of Bloch wave boundary conditions and we discuss relevant properties of the $S$ matrix. We consider the implications on the application of the formalism in conjunction with a cellular multiple scattering description of the electronic structure, and demonstrate the convergence properties concerning the angular momentum expansions.
\end{abstract}

DOI: 10.1103/PhysRevB.69.125104

PACS number(s): 72.10.Bg, 72.15.-v, 71.15.-m

\section{INTRODUCTION}

The study of transport properties of solids has always been a challenge for experimental and theoretical condensed matter physics. The difficulty lies among other things in the fact that the transport properties are by definition related to nonequilibrium situations, so that their modeling is not easily based on standard techniques. Fortunately, in the regime of linear response to weak external fields, the study can be based on ground state properties, by treating the field as a perturbation. That this approach is founded solidly is guaranteed by the fluctuation-dissipation theorem; in particular for the case of electrical conductivity this is expressed by the famous result of Kubo. ${ }^{1}$

In the past decades, the development of first-principles methods for the calculation of the electronic structure of solids has been accompanied by corresponding advances in the formalism and methods for the calculation of transport properties. Technological interest has given a push to the field, and novel effects such as the giant and the tunneling magnetoresistance (GMR and TMR) have been investigated theoretically and applied in technology. Furthermore, the size of today's electronic devices is so small that the understanding of ballistic (i.e., phase-coherent) transport has become important not only for basic physics but also for applications. As a result of these developments, ideas and methods that initially were conceived for the understanding of electronic transport in simple cases have been combined with techniques based on a realistic description of the electronic structure in order to give reliable and material-specific results. ${ }^{2}$ Without claiming to give a complete list, we mention that for diffusive transport through disordered systems there exist methods and results based on the combination of the Korringa, Kohn, and Rostoker (KKR) Green function method with the Boltzmann formalism, ${ }^{3-5}$ on the coherent potential approximation (CPA) combined with Kubo-Greenwood ${ }^{1,6}$ theory ${ }^{7-9}$ or on the tight-binding method. ${ }^{10}$ For ballistic transport there exist methods based, for example, on lowenergy electron diffraction (LEED) ${ }^{11,12}$ and layer $\mathrm{KKR}^{13}$ or similar layer-type ${ }^{14}$ techniques, on the tight-binding approach, ${ }^{15-17}$ and on the transfer matrix concept ${ }^{18,19}$ these mostly combine the Landauer-Büttiker approach ${ }^{20,21}$ with electronic structure methods, as will be done in the present paper. Moreover, due to potential applications in GMR and TMR devices, spin-dependent transport has come to the center of interest with an emphasis on conduction in magnetic multilayers $2,5,9,15,22-25$ and ferromagnet-semiconductor hybrides. ${ }^{13,16,26-31}$ In these systems the electronic spin degrees of freedom are accounted for in order to achieve spindependent resistance. In addition, novel systems such as nanowires or atomic-size contacts are created experimentally and demand interpretation of their transport properties.

In this paper we present a method for the calculation of ballistic transport from first principles, which combines the KKR ab initio Green-function technique with the Baranger and Stone ${ }^{32}$ formulation of the ballistic transport. The method is suitable for layered systems and interfaces with two-dimensional periodicity, as well as for atomic size constrictions connecting two leads or nanowires, ${ }^{33}$ and supports spin-dependent effects. Since the KKR technique in the screened formalism offers linear scaling of the computational effort with the number of layers, ${ }^{34}$ our method can handle large systems. Additionally we present the proof of some theorems concerning the transmission probability of wave packets in crystalline, rather than free-electron, environments.

The paper is organized as follows. In Sec. II we give a description of the setup of the physical systems that our method can be used for. In Sec. III we briefly address the approximations and assumptions made, also in connection to the Kubo-Greenwood and the Baranger-Stone formalism. Sections IV and V are devoted to making the connection to the Landauer-Büttiker formalism, and some elements of scattering theory are given there. The conductance formalism for the KKR method is developed in Sec. VI. Some examples illustrating the convergence properties of the method are given in Sec. VII. Finally, we conclude in Sec. VIII. The appendixes contain parts where lengthy mathematical manipulations were needed.

\section{SETUP OF THE PROBLEM}

The systems that we study consist of two half infinite perfect crystalline leads, left (L) and right (R), attached to a 


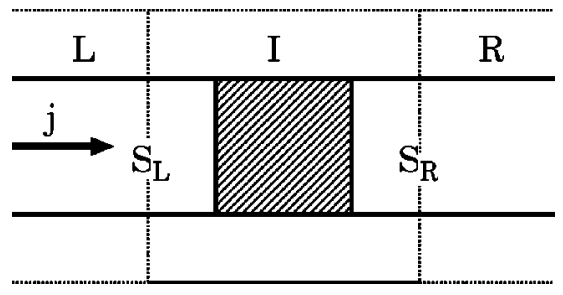

FIG. 1. Setup of the problem. The regions $\mathrm{L}$ and $\mathrm{R}$ correspond to perfect semi-infinite crystalline leads (not necessarily of the same material), attached to an "interaction region" I. This includes the interface or other structures sandwiched between L and R, plus a few monolayers of the leads at each side so that the evanescent interface states can decay. The conductance is evaluated between the surfaces $S_{\mathrm{L}}$ and $S_{\mathrm{R}}$, where only bulk Bloch states should exist.

slab that is considered as the "interaction" region (I); schematically this is represented in Fig. 1. The surfaces $S_{\mathrm{L}}$ and $S_{\mathrm{R}}$ should be chosen far enough within the leads so that any localized interface states have decayed and only Bloch states are present there. The direction of growth is taken to be the $z$ direction. We consider systems with a two-dimensional (2D) periodicity in the $x y$ plane where the scattering region is embedded between two semi-infinite crystalline leads. Moreover, summing up current contributions in real space we can calculate the conductance in the presence of defects in nanowires ${ }^{43,44}$ and we can even treat atomic sized constrictions between infinite leads if the current flow is localized in the constriction region, which is usually the case. ${ }^{45}$ Thus we can simulate transport through small molecules or a break junction geometry.

\section{KUBO FORMALISM}

The connection between the ballistic dc conductance or conductivity and the one-electron Green function in the linear-response regime has been given in the past. ${ }^{32,35-37} \mathrm{In}$ short, one uses first-order perturbation theory to calculate the effect of a weak, time-oscillating electric field on the density matrix of the system; then, the frequency of the oscillating field is taken to zero, and the dissipative term of the expectation value of the current gives the dc linear response of the system. The result is just an expression for the Kubo conductivity of the system at the limit of zero temperature. Integration by parts of the conductivity expression gives the relation among current, conductance, and voltage. Baranger and Stone ${ }^{32}$ have proven that the conductance is a Fermi-level property, although the two-point conductivity within the sample can also have contributions from other energy levels when magnetic fields are present. Here we briefly describe the relevant formalism, in connection to the rest of the paper.

The general relation between the current density at some point, $\mathbf{j}(\mathbf{r})$, and the electric field at all other points of a material, $\mathbf{E}\left(\mathbf{r}^{\prime}\right)$, in first-order perturbation theory for a stationary state, is given by

$$
\mathbf{j}(\mathbf{r})=\int d^{3} r^{\prime} \boldsymbol{\sigma}\left(\mathbf{r}, \mathbf{r}^{\prime}\right) \mathbf{E}\left(\mathbf{r}^{\prime}\right)
$$

The nonlocal conductivity tensor $\boldsymbol{\sigma}\left(\mathbf{r}, \mathbf{r}^{\prime}\right)$ is connected to the retarded one-electron Green function $G^{+}\left(\mathbf{r}, \mathbf{r}^{\prime} ; E\right)$ of the system via the famous Kubo-Greenwood result. Its frequently used form is ${ }^{7}$

$$
\begin{aligned}
\boldsymbol{\sigma}\left(\mathbf{r}, \mathbf{r}^{\prime}\right)= & -\frac{\hbar}{\pi} \int d E\left[-f^{\prime}(E)\right]\left(\frac{e \hbar}{m} \nabla \operatorname{Im} G^{+}\left(\mathbf{r}, \mathbf{r}^{\prime} ; E\right)\right) \\
& \times\left(\frac{e \hbar}{m} \nabla^{\prime} \operatorname{Im} G^{+}\left(\mathbf{r}^{\prime}, \mathbf{r} ; E\right)\right) .
\end{aligned}
$$

Here, $f^{\prime}(E)$ is the Fermi function derivative and Im denotes the imaginary part. In terms of the difference between the retarded and advanced Green function $G^{-}$,

$$
\begin{aligned}
\Delta G\left(\mathbf{r}, \mathbf{r}^{\prime} ; E\right) & :=G^{+}\left(\mathbf{r}, \mathbf{r}^{\prime} ; E\right)-G^{-}\left(\mathbf{r}, \mathbf{r}^{\prime} ; E\right) \\
& =-2 \pi i \int \operatorname{da}_{a}(\mathbf{r}) \psi_{a}^{*}\left(\mathbf{r}^{\prime}\right) \delta\left(E-E_{a}\right),
\end{aligned}
$$

the conductivity can be written

$$
\begin{aligned}
\boldsymbol{\sigma}\left(\mathbf{r}, \mathbf{r}^{\prime}\right)= & -\frac{e^{2} \hbar^{3}}{16 \pi m^{2}} \int d E\left[-f^{\prime}(E)\right] \\
& \times \Delta G\left(\mathbf{r}, \mathbf{r}^{\prime} ; E\right) \overleftrightarrow{\nabla} \overleftrightarrow{\nabla}^{\prime} \Delta G\left(\mathbf{r}^{\prime}, \mathbf{r} ; E\right),
\end{aligned}
$$

where the symbol $\overleftrightarrow{\nabla}$ is defined as $f(\mathbf{r}) \overleftrightarrow{\nabla} g(\mathbf{r})=f(\mathbf{r}) \nabla g(\mathbf{r})$ $-[\boldsymbol{\nabla} f(\mathbf{r})] g(\mathbf{r})$. Here only the $\delta$-function part of the Green function has been kept; the principal-value part gives rise to the reactive term, which is zero for dc conductance. ${ }^{32}$ Upon taking the limit of zero temperature, the derivative of the Fermi-Dirac function becomes a $\delta$ function according to $-f^{\prime}(E) \rightarrow \delta\left(E-E_{F}\right)$ and contributions to the conductivity only arise from the Fermi level.

In the absence of a magnetic field, as assumed in our work, the nonlocal conductivity tensor is symmetric under the interchange of $\mathbf{r}$ and $\mathbf{r}^{\prime}$, and divergenceless:

$$
\begin{gathered}
\boldsymbol{\sigma}\left(\mathbf{r}, \mathbf{r}^{\prime}\right)=\boldsymbol{\sigma}\left(\mathbf{r}^{\prime}, \mathbf{r}\right), \\
\boldsymbol{\nabla} \cdot \boldsymbol{\sigma}\left(\mathbf{r}, \mathbf{r}^{\prime}\right)=\boldsymbol{\sigma}\left(\mathbf{r}, \mathbf{r}^{\prime}\right) \cdot \dot{\boldsymbol{\nabla}}^{\prime}=0,
\end{gathered}
$$

where $\bar{\nabla}$ means that the operator acts to the function on its left. The former relation is a consequence of the symmetry $G\left(\mathbf{r}, \mathbf{r}^{\prime}\right)=G\left(\mathbf{r}^{\prime}, \mathbf{r}\right)$ of the Green function for local potentials. The latter is a result of current conservation in the absence of magnetic field; if a magnetic field is present, one has merely $\boldsymbol{\nabla} \cdot \boldsymbol{\sigma}\left(\mathbf{r}, \mathbf{r}^{\prime}\right) \cdot \overleftrightarrow{\boldsymbol{\nabla}}^{\prime}=0$ instead. ${ }^{32}$

In Eq. (1), of course, $\mathbf{E}\left(\mathbf{r}^{\prime}\right)$ is the total field, i.e., the external field plus the field being induced by charge relaxations; the electronic gas and nuclei must be also fully taken into account. This is not easy to handle; however, this expression can be integrated over the whole sample leaving only the current in relation to the applied voltage by exploiting the fact that $\mathbf{E}(\mathbf{r})=\boldsymbol{\nabla} V(\mathbf{r})$, where $V(\mathbf{r})$ is the electrostatic potential. Inserting this into Eq. (1) one gets for the total current $J$ through $S_{\mathrm{R}}$ 


$$
J:=\int_{S_{\mathrm{R}}} d S \hat{z} \hat{\mathbf{j}}(\mathbf{r})=\Delta V \int_{S_{\mathrm{R}}} d S \int_{S_{\mathrm{L}}} d S^{\prime} \hat{z} \boldsymbol{\sigma}\left(\mathbf{r}, \mathbf{r}^{\prime}\right) \hat{z}^{\prime},
$$

after an integration by parts and use of Eq. (7) (both $\hat{z}$ and $\hat{z}^{\prime}$ are pointing outwards). Here $\Delta V$ is the external bias voltage. The "zero-current theorem" stating that the ground-state electrostatic potential gives always zero current density ${ }^{38}$ has been used, so only the externally applied voltage $\Delta V$ remains. Thus one can recognize the conductance as the flux of the conductivity tensor through the surfaces $S_{\mathrm{L}}$ and $S_{\mathrm{R}}$ :

$$
g=\int_{S_{\mathrm{L}}} d S \int_{S_{\mathrm{R}}} d S^{\prime} \hat{z} \boldsymbol{\sigma}\left(\mathbf{r}, \mathbf{r}^{\prime}\right) \hat{z}^{\prime} .
$$

Substituting Eq. (5) one obtains for the conductivity:

$$
\begin{aligned}
g= & -\frac{e^{2} \hbar^{3}}{8 \pi m^{2}} \int_{S_{\mathrm{L}}} d S \int_{S_{\mathrm{R}}} d S^{\prime} G^{+}\left(\mathbf{r}, \mathbf{r}^{\prime} ; E_{F}\right) \vec{\nabla}_{z} \overleftrightarrow{\mathbf{\nabla}}_{z}^{\prime} \\
& \times G^{-}\left(\mathbf{r}^{\prime}, \mathbf{r} ; E_{F}\right),
\end{aligned}
$$

where the terms involving $G^{+} \overleftrightarrow{\mathbf{\nabla}}_{z} \overleftrightarrow{\mathbf{\nabla}}_{z}^{\prime} G^{+}$and $G^{-} \overleftrightarrow{\mathbf{\nabla}}_{z} \overleftrightarrow{\mathbf{\nabla}}_{z}^{\prime} G^{-}$ vanish. $^{32}$

\section{LANDAUER FORMALISM}

In the Landauer approach, the conductance problem is viewed from the aspect of scattering theory. In this way, the causal relation between voltage and current is conceptually reversed. ${ }^{39}$ Instead of applying a voltage and examining the current as the response, a current is forced to flow through the sample and the voltage is viewed as the result of the pileup of carriers at the various obstacles, forming residual resistivity dipoles. The result is, of course, again the usual current-voltage relation. In a multilead experiment, if each lead $n$ is held in potential $V_{n}$, then the current $J_{n}$ going out through this lead is

$$
J_{n}=\sum_{m \neq n} g_{n m} V_{m}
$$

The coefficients $g_{n m}$ describe the conductance of the system, and in the case of only two leads there is only one conductance coefficient $g$.

The concept of incoming and outgoing scattering channels is introduced, which play the same role as in and out states in scattering theory; in the cases of our interest they are Bloch states in the leads. We need to describe the scattering process of one-electron Bloch states incident from lead $\mathrm{L}$ as incoming waves and scattered into lead $\mathrm{L}$ or $\mathrm{R}$ as outgoing waves; thus an $S$-matrix formulation is appropriate. One has waveguide or crystalline geometry in the leads, rather than the free-space geometry of usual scattering theory, and in addition the leads can consist of different materials. In this respect, the usual scattering theory needs a few modifications to be applicable.

Once the $S$-matrix elements are available, one can readily calculate the transmission probability $T_{n f ; m i}$ from each incoming channel $i$ in lead $m$ to each outgoing $f$ in lead $n$, and use the Landauer-Büttiker formula to calculate the conductance $g_{n m}$ from lead $m$ to lead $n:{ }^{21}$

$$
g_{n m}=\frac{e^{2}}{h} \sum_{f i} T_{n f ; m i}=\frac{e^{2}}{h} \sum_{f i}\left|S_{n f ; m i}\right|^{2} .
$$

Stone and Szafer ${ }^{35}$ and Baranger and Stone ${ }^{32}$ showed the connection between the Landauer-Büttiker formula with the Kubo result starting from perturbation theory, considering free-electron leads. In Sec. V we shall follow their analysis closely, but prove some of the theorems needed for crystalline leads taking into account the Bloch character of the incoming and outgoing channels.

The $S$-matrix formulation in such problems and the question of unitarity will be shortly addressed now. First of all, the in states and out states, which in a usual formulation are plane or spherical waves, are here propagating Bloch states in the leads. Whether such a state $\Psi_{\mathbf{k}}$ is incoming or outgoing is determined not by the Bloch wave vector $\mathbf{k}$, but by the group velocity $\mathbf{v}_{\mathbf{k}}=\nabla_{\mathbf{k}} E_{\mathbf{k}}$. Suppose that a particular lead is grown in the $z$ direction, with the unit vector $\hat{z}$ pointing away from the sample. Then, the Bloch state is outgoing if $\left(\mathbf{v}_{\mathbf{k}}\right)_{z}$ $>0$, and incoming if $\left(\mathbf{v}_{\mathbf{k}}\right)_{z}<0$. Evidently the set of in states can be different from the set of out states. Consider, for example, the case of only two leads of different materials, say $\mathrm{L}$ in the left with wave functions $\Psi^{\mathrm{L}}$ and $\mathrm{R}$ in the right with wave functions $\Psi^{\mathrm{R}}$, sandwiching the interaction region I. The set of in states for this problem will consist of righttraveling waves $\Psi_{\text {in }}^{\mathrm{L}}$ and left-traveling $\Psi_{\text {in }}^{\mathrm{R}}$, while the set of out states will consist of left-traveling waves $\Psi_{\text {out }}^{\mathrm{L}}$ and righttraveling waves $\Psi_{\text {out }}^{\mathrm{R}}$.

The total wave function in the system will be asymptotically a linear combination of in and out states; in particular, if we choose incidence from lead L as a boundary condition, we have the form

$$
\Psi_{\mathbf{k} a}^{\mathrm{tot}}(\mathbf{r})= \begin{cases}\Psi_{\mathbf{k} a}^{\mathrm{Lin}}+\sum_{\mathbf{k}^{\prime} a^{\prime}} r_{\mathbf{k} a \mathbf{k}^{\prime} a^{\prime}} \Psi_{\mathbf{k}^{\prime} a^{\prime}}^{\mathrm{L} \text { out }}, & z \rightarrow-\infty \\ \sum_{\mathbf{k}^{\prime} a^{\prime}} t_{\mathbf{k} a \mathbf{k}^{\prime} a^{\prime}} \Psi_{\mathbf{k}^{\prime} a^{\prime}}^{\mathrm{R} \text { out }}, & z \rightarrow+\infty .\end{cases}
$$

For finite $z$ values also evanescent states have to be included in the summations; however, they die out for $z \rightarrow \pm \infty$. Here, band indices $a$ and $a^{\prime}$ and Bloch wave vectors $\mathbf{k}$ and $\mathbf{k}^{\prime}$, have been introduced; elastic scattering is implied. The total wave function $\Psi_{\mathbf{k} a}^{\text {tot }}(\mathbf{r})$ is characterized by $\mathbf{k} a$ in the sense of the boundary condition, i.e., it originates from an in state that has this wave vector. The transmission amplitude $t_{\mathbf{k} a \mathbf{k}^{\prime} a^{\prime}}$ and the reflection amplitude $r_{\mathbf{k} a \mathbf{k}^{\prime} a^{\prime}}$ are related to the elements of the $S$ matrix of the system.

The normalization of the in and out states determines whether these amplitudes $t$ and $r$ are identical with the $S$-matrix elements or not, since the $S$ matrix must always be unitary. If one normalizes the in and out states to unit flux (as $\Psi \rightarrow \Psi / \sqrt{v}$ ), then the transmission probability from state $\Psi_{\mathbf{k} a \text { in }}^{\mathrm{L}}$ to state $\Psi_{\mathbf{k}^{\prime} a^{\prime} \text { out }}^{\mathrm{R}}$ is just $T_{\mathrm{R} \mathbf{k}^{\prime} a^{\prime} ; \mathrm{L} \mathbf{k} a}=\left|t_{\mathbf{k} a \mathbf{k}^{\prime} a^{\prime}}\right|^{2}$ $=\left|S_{\mathbf{k} a \mathbf{k}^{\prime} a^{\prime}}\right|^{2}$. But with the usual normalization to unit prob- 
ability in space, the transmission probability must invoke the group velocities to account for the different flux of in and out states: ${ }^{40}$

$$
T_{\mathrm{R} \mathbf{k}^{\prime} a^{\prime} ; \mathrm{L} \mathbf{k} a}=\left|S_{\mathbf{k} a \mathbf{k}^{\prime} a^{\prime}}\right|^{2}=\left|t_{\mathbf{k} a \mathbf{k}^{\prime} a^{\prime}}\right|^{2} \frac{\left|\left(v_{\mathbf{k}^{\prime} a^{\prime}}^{\text {out }}\right)_{z}\right|}{\left|\left(v_{\mathbf{k} a}^{\text {in }}\right)_{z}\right|} .
$$

This point will be addressed in detail in the following section.

\section{CONNECTION BETWEEN KUBO AND LANDAUER APPROACHES}

The connection of the Landauer approach involving the $S$ matrix to the Kubo-Greenwood conductivity formula involving the Green functions has been given by Fisher and Lee, ${ }^{36}$ Stone and Szafer, ${ }^{35}$ and Baranger and Stone ${ }^{32}$ in the case of free electrons in the leads. However, for crystalline leads one must account for the relevant band structure. Here we present some proofs needed to extend the above results to crystalline leads. In particular we shall pursue the $S$-matrix elements between in states from L leads and out states in $\mathrm{R}$ leads and see how these connect to the Green function of the system and to the conductance. In this way the connection of the Kubo to the Landauer formalism will be made. We proceed in three steps: (i) we find an expression for the transmission amplitude $t_{\mathbf{k} a ; \mathbf{k}^{\prime} a^{\prime}}$; (ii) we express the asymptotic Green function in terms of the $S$ matrix; and (iii) we express the $S$ matrix in terms of $t_{\mathbf{k} a: \mathbf{k}^{\prime} a^{\prime}}$.

(i) We start with the calculation of current matrix elements between Bloch states. In particular, let $\Psi_{\mathbf{k} a}^{\text {in }}$ and $\Psi_{\mathbf{k} a}^{\text {out }}$ be incoming and outgoing Bloch states (in the sense described before, i.e., right and left traveling) of the same energy $\left(E_{\mathbf{k} a}=E_{\mathbf{k}^{\prime} a^{\prime}}\right)$ in the same lead; the states are normalized to unit probability rather than unit flux. Also, let $S$ be the lead cross section, normal to the $z$ direction. Then the following orthogonality relations hold for the current matrix elements: ${ }^{41}$

$$
\begin{aligned}
\int_{S} d S \Psi_{\mathbf{k} a}^{\text {in } * \overleftrightarrow{\nabla}_{z}} \Psi_{\mathbf{k}^{\prime} a^{\prime}}^{\text {in }} & =\int_{S} d S \Psi_{\mathbf{k} a}^{\text {out } * \overleftrightarrow{\nabla}_{z}} \Psi_{\mathbf{k}^{\prime} a^{\prime}}^{\text {out }} \\
& =i \frac{2 m}{\hbar}\left(v_{\mathbf{k} a}\right)_{z} \delta_{\mathbf{k k}^{\prime}} \delta_{a a^{\prime}} \\
\int_{S} d S \Psi_{\mathbf{k} a}^{\text {in } * \overleftrightarrow{\nabla}_{z}} \Psi_{\mathbf{k}^{\prime} a^{\prime}}^{\text {out }} & =0 \quad\left(\text { for } E_{\mathbf{k}^{\prime} a^{\prime}}=E_{\mathbf{k} a}\right)
\end{aligned}
$$

( $\Psi_{\mathbf{k} a}^{\mathrm{in}}$ and $\Psi_{\mathbf{k}^{\prime} a^{\prime}}^{\text {out }}$ here are supposed to be left- and righttraveling states in the same lead). These have been proven before $^{32}$ for free electrons; we present a proof for Bloch states in Appendix A.

Equations (15) and (16) can be used to project a scattering wave function onto a particular channel; using them in connection with Eq. (13) we can extract the transmission amplitude out of $\Psi_{\mathbf{k} a}^{\text {tot }}$ :

$$
t_{\mathbf{k} a ; \mathbf{k}^{\prime} a^{\prime}} i \frac{2 m}{\hbar}\left(v_{\mathbf{k}^{\prime} a^{\prime}}^{\mathrm{out}}\right)_{z}=\int_{S} d S \Psi_{\mathbf{k} a}^{\mathrm{tot} *}(\mathbf{r}) \overleftrightarrow{\nabla}_{z} \Psi_{\mathbf{k}^{\prime} a^{\prime}}^{\mathrm{R} \text { out }}(\mathbf{r}) .
$$

(ii) Next we consider the asymptotic expression for the retarded Green function $G^{+}\left(\mathbf{r}, \mathbf{r}^{\prime} ; E\right)$, with $\mathbf{r}$ and $\mathbf{r}^{\prime}$ in different leads and going to infinity. This can be found ${ }^{32}$ by using the boundary condition that $G^{+}\left(\mathbf{r}, \mathbf{r}^{\prime} ; E\right)$ should represent an outgoing wave at $\mathbf{r}, G^{-}\left(\mathbf{r}, \mathbf{r}^{\prime} ; E\right)$ should represent an incoming wave at $\mathbf{r}$, and

$$
G^{+}\left(\mathbf{r}, \mathbf{r}^{\prime} ; E\right)=G^{-*}\left(\mathbf{r}^{\prime}, \mathbf{r} ; E\right)
$$

The Green function then, expanded in in and out states, has the asymptotic form

$$
\begin{aligned}
G^{+}\left(\mathbf{r}, \mathbf{r}^{\prime} ; E\right)= & \sum_{\mathbf{k} a} \sum_{\mathbf{k}^{\prime} a^{\prime}} A_{\mathbf{k} a ; \mathbf{k}^{\prime} a^{\prime}} \Psi_{\mathbf{k} a}^{\mathrm{R} \text { out }}(\mathbf{r}) \Psi_{\mathbf{k}^{\prime} a^{\prime}}^{\mathrm{Lin} *}\left(\mathbf{r}^{\prime}\right) \\
& \left(\text { for } z \rightarrow \infty, z^{\prime} \rightarrow-\infty\right) .
\end{aligned}
$$

We are now seeking a relation between the coefficients $A_{\mathbf{k} a ; \mathbf{k}^{\prime} a^{\prime}}$ and the transmission amplitudes $t_{\mathbf{k} a ; \mathbf{k}^{\prime} a^{\prime}}$. Equation (17) was used together with the Lippmann-Schwinger equation and Eq. (10) in Ref. 32 to prove this for free-electron leads. However, the Lippmann-Schwinger equation is difficult to handle when we have different materials in the leads, because it connects the Bloch wave functions of different materials in the infinite leads, thus there is no localized perturbation. Alternatively, one can start directly from the definition $^{42}$ of the $S$ matrix in terms of the time-dependent retarded Green function (propagator), looking at the transmission amplitude from an initial wave packet $\Phi_{i}^{\mathrm{L} \text { in }}(\mathbf{r}, t)$ $=\int d E_{i} e^{-i E_{i} t} a_{i}\left(E_{i}\right) \Psi_{i}^{\mathrm{L} \text { in }}\left(\mathbf{r} ; E_{i}\right)$, incident from the left, to a final one $\Phi_{f}^{\mathrm{R} \text { out }}(\mathbf{r}, t)=\int d E_{f} e^{-i E_{f} t} a_{f}\left(E_{f}\right) \Psi_{f}^{\mathrm{R} \text { out }}\left(\mathbf{r} ; E_{f}\right)$, outgoing to the right. Here $\Psi_{i}$ and $\Psi_{f}$ denote itinerant Bloch states and we have propagated the initial and final states to $t$ and $t^{\prime}$ by their corresponding bulk Hamiltonians, giving the exponential factors. At the end $a_{i}$ and $a_{f}$ will be taken as extremely peaked distributions around the same energy $E_{i} ; i$ and $f$ represent then definite $\mathbf{k}^{\prime} a^{\prime}$ and $\mathbf{k} a$. (Atomic units with $\hbar=1$ are implied.) One gets

$$
\begin{aligned}
& S_{f i}:=\lim _{t \rightarrow \infty} \int d^{3} r \int d^{3} r^{\prime} \Phi_{f}^{\mathrm{R} \text { out } *}(\mathbf{r}, t) \\
& t^{\prime} \rightarrow-\infty \\
& \times G^{+}\left(\mathbf{r}, t ; \mathbf{r}^{\prime}, t^{\prime}\right) \Phi_{i}^{\mathrm{Lin}}\left(\mathbf{r}^{\prime}, t^{\prime}\right) \\
& =\lim _{t \rightarrow \infty} \int d^{3} r \int d^{3} r^{\prime} \Phi_{f}^{\mathrm{R} \text { out } *}(\mathbf{r}, t) \int d E e^{-i E\left(t-t^{\prime}\right)} \\
& t^{\prime} \rightarrow-\infty \\
& \times G^{+}\left(\mathbf{r}, \mathbf{r}^{\prime} ; E\right) \Phi_{i}^{\mathrm{Lin}}\left(\mathbf{r}^{\prime}, t^{\prime}\right) \\
& =\lim _{\substack{t \rightarrow \infty \\
t^{\prime} \rightarrow-\infty}} \int d E e^{-i E\left(t-t^{\prime}\right)} \sum_{f^{\prime} i^{\prime}} A_{f^{\prime} i^{\prime}}(E) \int d E_{f} e^{i E_{f^{t}} a_{f}^{*}\left(E_{f}\right)} \\
& \times\left(\Psi_{f}^{\mathrm{R} \text { out }}\left(E_{f}\right), \Psi_{f^{\prime}}^{\mathrm{R} \text { out }}(E)\right) \int d E_{i} e^{-i E_{i} t^{\prime}} a_{i}\left(E_{i}\right) \\
& \times\left(\Psi_{i^{\prime}}^{\mathrm{Lin}}(E), \Psi_{i}^{\mathrm{L} \text { in }}\left(E_{i}\right)\right) \\
& =\int d E a_{f}^{*}(E) a_{i}(E) v_{i} v_{f} A_{f i}(E) \\
& =A_{f i}\left(E_{i}\right) \sqrt{v_{i} v_{f}} \quad \text { (on shell). }
\end{aligned}
$$


The velocities appearing represent the $z$ component of the group velocity, since in waveguide geometry the $\mathbf{k}_{\|}$'s form in reality a very dense discrete set so that the wave packet is constructed for a definite $\mathbf{k}_{\|}$from the continuous $k_{z}$ spectrum. Having this in mind, we have used in the derivation of Eq. (20) the orthonormality condition for Bloch waves

$$
\begin{aligned}
\left(\Psi_{\mathbf{k} a}\left(E_{f}\right), \Psi_{\mathbf{k}^{\prime} a^{\prime}}(E)\right) & :=\int d^{3} r \Psi_{\mathbf{k} a}^{*}\left(\mathbf{r}, E_{f}\right) \Psi_{\mathbf{k}^{\prime} a^{\prime}}(\mathbf{r} ; E) \\
& =\delta_{\mathbf{k}_{\|} \mathbf{k}_{\|}^{\prime}} \delta_{a a^{\prime}} \delta\left(k_{z}-k_{z}^{\prime}\right) \\
& =\delta_{\mathbf{k}_{\|} \mathbf{k}_{\mid}^{\prime}} \delta_{a a^{\prime}} v_{z} \delta\left(E-E_{f}\right) .
\end{aligned}
$$

In addition we have used the normalization of wave packets to unit probability

$$
\begin{aligned}
1 & =\int d E a^{*}(E) \int d E^{\prime} a\left(E^{\prime}\right)\left(\Psi(E), \Psi\left(E^{\prime}\right)\right) \\
& =\int d E|a(E)|^{2} v_{E},
\end{aligned}
$$

which for a very peaked distribution $a(E)$ around some energy $E_{i}$ gives

$$
\int d E|a(E)|^{2}=1 / v_{E_{i}},
$$

implying that in the limiting case $|a(E)| \rightarrow \sqrt{\delta\left(E-E_{i}\right) / v_{E_{i}}}$. Finally, in the last step we have assumed that $a_{f}(E)$ and $a_{i}(E)$ are both peaked around the same energy $E_{i}$ so that the wave packet goes to a single Bloch function, whence $a_{f}^{*}(E) a_{i}(E) \rightarrow \delta\left(E-E_{i}\right) / \sqrt{v_{f}\left(E_{i}\right) v_{i}\left(E_{i}\right)}$. Thus, the final result (21) is valid for on-energy-shell scattering of Bloch waves; otherwise, for general wave packets, Eq. (20) must be applied. Working with wave packets has guaranteed the correct normalization.

From Eq. (21) we see that the coefficients in the Green function asymptotic expansion are just $S$-matrix elements (normalized to the group velocities). In this form, the $S$ matrix is unitary, i.e., the scattering probability is

$$
T_{f i}=\left|S_{f i}\right|^{2} .
$$

(iii) Finally we show that the relation of the $S$ matrix to the previously defined transmission amplitude of Eq. (13) is

$$
S_{f i}=t_{f i} \frac{\sqrt{v_{f}}}{\sqrt{v_{i}}}=A_{f i} \sqrt{v_{f} v_{i}},
$$

i.e., a normalization involving the group velocities is needed. This can be seen by noting that an incoming wave packet $\Phi_{\text {in }}=\int d E a(E) \Psi_{\text {in }}(E)$, normalized to unit probability as in Eq. (23), evolves partly into the wave packet $\Phi_{\text {out }}$ $=\int \operatorname{dEt}(E) a(E) \Psi_{\text {out }}(E)$ according to Eq. (13). Assuming that $a(E)$ is so much peaked around $E_{0}$ that $t$ and $v$ are constant in this energy range, the scattering probability is given by the normalization factor of the outgoing wave packet:

$$
\begin{aligned}
T_{f i} & =|| \Phi_{\text {out }}||^{2} \\
& =|t|^{2} \int d E \int d E^{\prime}|a(E)|^{2}\left(\Psi_{\text {out }}(E), \Psi_{\text {out }}\left(E^{\prime}\right)\right) \\
& =|t|^{2} \int d E|a(E)|^{2} \int d E^{\prime} \delta\left(E-E^{\prime}\right) v_{\text {out }}=|t|^{2} v_{\text {out }} / v_{\text {in }},
\end{aligned}
$$

where the normalizations (22) and (24) have been utilized. Comparing this to Eq. (25) proves Eq. (26) up to a phase factor, which can be seen to be just unity by creating a wave packet out of $\Psi^{\text {tot }}$ in Eq. (13) and constructing the $S$-matrix element. Furthermore the expression (14) results from Eqs. (25) and (26).

Combining all the above, we may rewrite Eq. (19) as

$$
\begin{aligned}
G^{+}\left(\mathbf{r}, \mathbf{r}^{\prime} ; E\right) & =\sum_{\mathbf{k} a} \sum_{\mathbf{k}^{\prime} a^{\prime}} \frac{S_{\mathbf{k} a ; \mathbf{k}^{\prime} a^{\prime}}}{\sqrt{\left(v_{\mathbf{k}^{\prime} a^{\prime}}\right)_{z}\left(v_{\mathbf{k} a}\right)_{z}}} \Psi_{\mathbf{k}^{\prime} a^{\prime}}^{\mathrm{Lin} *}\left(\mathbf{r}^{\prime}\right) \Psi_{\mathbf{k} a}^{\mathrm{Rout}}(\mathbf{r}) \\
& =\sum_{\mathbf{k} a} \sum_{\mathbf{k}^{\prime} a^{\prime}} \frac{t_{\mathbf{k} a ; \mathbf{k}^{\prime} a^{\prime}}}{\left(v_{\mathbf{k}^{\prime} a^{\prime}}\right)_{z}} \Psi_{\mathbf{k}^{\prime} a^{\prime}}^{\mathrm{Lin} *}\left(\mathbf{r}^{\prime}\right) \Psi_{\mathbf{k} a}^{\mathrm{Rout}}(\mathbf{r})
\end{aligned}
$$

which gives us for the advanced Green function

$$
G^{-}\left(\mathbf{r}, \mathbf{r}^{\prime} ; E\right)=\sum_{\mathbf{k} a} \sum_{\mathbf{k}^{\prime} a^{\prime}} \frac{t_{\mathbf{k} a ; \mathbf{k}^{\prime} a^{\prime}}^{*}}{\left(v_{\mathbf{k}^{\prime} a^{\prime}}\right)_{z}} \Psi_{\mathbf{k}^{\prime} a^{\prime}}^{\mathrm{Rout} *}\left(\mathbf{r}^{\prime}\right) \Psi_{\mathbf{k} a}^{\mathrm{Lin}}(\mathbf{r}) .
$$

We can extract the $S$-matrix elements from the Green function:

$$
\begin{aligned}
S_{\mathbf{k} a ; \mathbf{k}^{\prime} a^{\prime}}= & \frac{\hbar^{3}}{4 m^{2}} \frac{1}{\sqrt{\left(v_{\mathbf{k}^{\prime} a^{\prime}}\right)_{z}\left(v_{\mathbf{k} a}\right)_{z}}} \int_{S_{L}} d S^{\prime} \\
& \times \int_{S_{R}} d S \Psi_{\mathbf{k}^{\prime} a^{\prime}}^{\mathrm{Lin}}\left(\mathbf{r}^{\prime}\right) \overleftrightarrow{\nabla}_{z}^{\prime} G^{+}\left(\mathbf{r}, \mathbf{r}^{\prime} ; E\right) \overleftrightarrow{\nabla}_{z} \Psi_{\mathbf{k} a}^{\mathrm{Rout} *}(\mathbf{r}),
\end{aligned}
$$

where $S_{L}$ and $S_{R}$ have to be sufficiently far apart so that the asymptotic formula (20) without evanescent states can be used (the evanescent states are always included in the Green function).

As a final result, we combine the above steps (i)-(iii) to arrive at the Landauer formula. We start from the BarangerStone expression (10), expand the Green functions according to Eqs. (27) and (28), and use the orthogonality relations (15) to get rid of some terms:

$$
\begin{aligned}
g & =-\frac{e^{2} \hbar^{3}}{8 \pi m^{2}} \int_{S_{\mathrm{L}}} d S \int_{S_{\mathrm{R}}} d S^{\prime} G^{+}\left(\mathbf{r}, \mathbf{r}^{\prime} ; E_{F}\right) \overleftrightarrow{\nabla}_{z} \overleftrightarrow{\nabla}_{z}^{\prime} G^{-}\left(\mathbf{r}^{\prime}, \mathbf{r} ; E_{F}\right) \\
& =\frac{e^{2}}{h} \sum_{\mathbf{k} a \text { in }} \sum_{\mathbf{k}^{\prime} a^{\prime} \text { out }}\left|t_{\mathbf{k} a ; \mathbf{k}^{\prime} a^{\prime}}\right|^{2} \frac{\left|\left(v_{\mathbf{k}^{\prime} a^{\prime}}\right)_{z}\right|}{\left|\left(v_{\mathbf{k} a}\right)_{z}\right|} \\
& =\frac{e^{2}}{h} \sum_{\mathbf{k} a \text { in }} \sum_{\mathbf{k}^{\prime} a^{\prime} \text { out }} T_{\mathbf{k}^{\prime} a^{\prime} ; \mathbf{k} a} .
\end{aligned}
$$




\section{THE CONDUCTANCE FORMULA IN THE KKR METHOD}

\section{A. The Green function in the KKR formalism}

In the KKR method one divides the crystal in atomic cells, with the atoms positioned at the sites $\mathbf{R}_{n}$, so that the crystal potential $V$ is expressed in each cell as $V_{n}(\mathbf{r})$ $=V\left(\mathbf{R}_{n}+\mathbf{r}\right)$. Then the one-electron retarded Green function is expanded in terms of local orbitals centered at the atomic sites $\mathbf{R}_{n}$ as

$$
\begin{aligned}
G^{+}\left(\mathbf{R}_{n}\right. & \left.+\mathbf{r}, \mathbf{R}_{n^{\prime}}+\mathbf{r}^{\prime} ; E\right) \\
= & -i \sqrt{E} \sum_{L} R_{L}^{n}\left(\mathbf{r}_{<} ; E\right) H_{L}^{n}\left(\mathbf{r}_{>} ; E\right) \delta_{n n^{\prime}} \\
& +\sum_{L L^{\prime}} R_{L}^{n}(\mathbf{r} ; E) G_{L L^{\prime}}^{n n^{\prime}}(E) R_{L}^{n^{\prime}}\left(\mathbf{r}^{\prime} ; E\right) .
\end{aligned}
$$

Here, $R_{L}^{n}(\mathbf{r} ; E)$ and $H_{L}^{n}(\mathbf{r} ; E)$ are, respectively, the regular and irregular solutions of the Schrödinger equation for the single potential $V_{n}(\mathbf{r})$ of the $n$th cell in free space. Atomic units are used $(e=-\sqrt{2}, \hbar=1, m=1 / 2)$. The index $L$ $=(l, m)$ represents the angular momentum quantum number. The position vector $\mathbf{r}$ is confined in the atomic cell $n ; \mathbf{r}_{>}$and $\mathbf{r}_{<}$are the longer and shorter, respectively, of $\mathbf{r}$ and $\mathbf{r}^{\prime}$. In Eq. (31) the first term gives the on-site contribution to the Green function, while the second is the so-called backscattering term, where the information on the intersite electron propagation is contained in the structure constants $G_{L L^{\prime}}^{n n^{\prime}}(E)$. These are related to the known structure constants of a reference system, e.g., vacuum, via an algebraic Dyson equation that includes the local $t$ matrix of each single-site potential. For further details we refer to Refs. 34.

The layered systems that shall be considered with the KKR formalism consist of two half-infinite crystalline leads, assumed to have perfect periodicity otherwise. Sandwiched between these leads is an "interaction" region where a different material can be placed and where the scattering of the Bloch waves takes place. Three cases can be handled in this respect: (i) systems with in-plane periodicity; ${ }^{30}$ (ii) wirelike structures embedded in vacuum (or in some other nonconducting medium) ${ }^{43,44}$ and (iii) atomic constrictions between semi-infinite leads.

Concerning case (i), when we consider systems with twodimensional in-plane $(x-y)$ periodicity (perpendicular to the direction of growth $z$ ), the interaction region and the two leads have common in-plane Bravais vectors. If needed, larger (nonprimitive) two-dimensional unit cells are taken to match the lattice constants of both materials; this is the case, for example, in an Fe/GaAs contact. ${ }^{30}$ The two-dimensional periodicity of the layered systems allows to Fourier transform the Green function in the $x$ and $y$ directions, obtaining a two-dimensional Bloch vector $\mathbf{k}_{\|}=\left(k_{x}, k_{y}\right)$ as a good quantum number, and retaining an index $i$ to characterize the layer in the direction of growth $z$. The Green function connecting the layers $i$ in the left lead and $i^{\prime}$ in the right lead is then written

$$
\begin{aligned}
G^{+} & \left(\mathbf{R}_{i}+\chi_{\nu}+\mathbf{r}, \mathbf{R}_{i^{\prime}}+\chi_{\nu^{\prime}}+\mathbf{r}^{\prime} ; E\right) \\
= & \frac{1}{S_{\mathrm{SBZ}}} \int_{\mathrm{SBZ}} d^{2} k_{\|} e^{i \mathbf{k}_{\|}\left(\chi_{\nu}-\chi_{\nu^{\prime}}\right)} \\
& \quad \times \sum_{L L^{\prime}} R_{L}^{i}(\mathbf{r} ; E) G_{L L^{\prime}}^{i i^{\prime}}\left(\mathbf{k}_{\|} ; E\right) R_{L^{\prime}}^{i^{\prime}}\left(\mathbf{r}^{\prime} ; E\right)
\end{aligned}
$$

with

$$
G_{L L^{\prime}}^{i i^{\prime}}\left(\mathbf{k}_{\|} ; E\right)=\sum_{\nu^{\prime}} e^{-i \mathbf{k}_{\|}\left(\chi_{\nu}-\chi_{\nu^{\prime}}\right)} G_{L L^{\prime}}^{i \nu ; i^{\prime} \nu^{\prime}}(E),
$$

where $\boldsymbol{\chi}_{\boldsymbol{\nu}}$ and $\boldsymbol{\chi}_{\boldsymbol{\nu}^{\prime}}$ are in-plane lattice vectors, $\mathbf{R}_{i}$ is the interlayer lattice vector, and SBZ is the surface Brillouin zone of the system and $S_{\mathrm{SBZ}}$ its area. $G_{L L^{\prime}}^{i i^{\prime}}\left(\mathbf{k}_{\|} ; E\right)$ are the $\mathbf{k}_{\|}$-dependent structure constants. In this equation each layer $i$ is assumed to have a unique atom type, hence only the index $i$ suffices to characterize the local wave function. In the case of more inequivalent atoms per layer, an extra index $\mu$ can be introduced to account for the propagation between different kinds of atoms. In the case of spin magnetism, the Green function is different for each spin direction $\sigma=\uparrow$ or $\downarrow$. The formalism can be generalized for fully relativistic calculations, where the spin-orbit coupling results in a mixing of the two spin channels.

Once Eq. (31) or Eq. (32) is substituted into the expression (10), with $\mathbf{r}$ and $\mathbf{r}^{\prime}$ in different leads, the on-site term of the Green function does not contribute, and only the intersite term survives; moreover, the spacial derivative affects only the local orbital functions $R_{L}^{n}(\mathbf{r})$ and $R_{L}^{n^{\prime}}(\mathbf{r})$, leaving the structure constants untouched. Finally, if Eq. (32) is used, the conductance appears at first $\mathbf{k}_{\|}$-resolved, $g\left(\mathbf{k}_{\|}\right)$, which is most convenient in structures with two-dimensional periodicity. A $\mathbf{k}_{\|}$integration then gives the result

$$
g=\frac{1}{S_{\mathrm{SBZ}}} \int_{\mathrm{SBZ}} d^{2} k_{\|} g\left(\mathbf{k}_{\|}\right) .
$$

In Eq. (10), both the retarded and the advanced Green functions are needed; however, they are related through the identity (18) for real $E$. This is used in our formulation; the energy $E$ is identified in the calculations with the Fermi level $E_{F}$ plus an (in principle infinitesimal) imaginary part $\epsilon$, which we take very small.

The case (ii) of wirelike structures is completely analogous, but the Fourier transform of Eq. (32) is not performed. More specifically, consider a wire embedded in vacuum (see also Fig. 2); the vacuum region will be also divided artificially in volume-filling cells. One can also consider defects within the wire, or even two wires attached to some cluster of atoms (as indicated in Fig. 2), and solve self-consistently for the electronic structure. Due to electronic states at the surface of the wire, the first one or two vacuum layers above the wire surface can contribute to the conductance, but after that the cross section can be truncated. The cross sections left and right, where the conductivity tensor must be evaluated, 

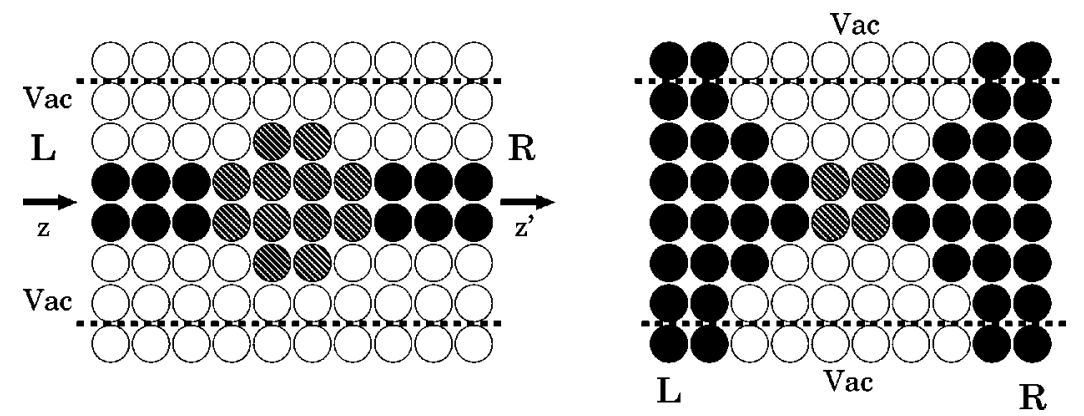

FIG. 2. Left: Setup for the calculation of conductance in a nanowire configuration. The filled circles represent the wire atoms (leads), including defects or an atomic cluster (shaded circles). The white circles represent the vacuum region. The conductance calculation is truncated at the dashed lines, assuming that the states of the nanowire have decayed beyond this region. The arrows labeled $z$ and $z^{\prime}$ represent the directions of growth of the two leads. Right: Similar setup for the calculation in the case of nanosize constrictions between infinite leads. Again a truncation at the dashed lines is taken, assuming that outside this region there is insignificant tunneling between the leads.

consist always of more than one atomic cell, since one must include the vacuum region. In this way the Green function must be considered for all combinations between cells on the left and the right, and the expression (10) for the conductance splits up in partial contributions corresponding to these combinations:

$$
g=\sum_{\mu^{\prime}(\text { left })} \sum_{\mu(\text { right })} g_{\mu \mu^{\prime}}
$$

with $g_{\mu \mu^{\prime}}$ given by Eq. (10) but integrated over the cross section of single atomic cells $\mu^{\prime}$ (left) and $\mu$ (right).

Analogously, in case (iii) a similar setup can be used if we consider semi-infinite 2D leads but current flow localized in space, as in the case of transport through a constriction. In this case we consider two planes as shown in Fig. 2 (right), while convergence must be checked with respect to the size of the regions considered in the summations of Eq. (34).

In the next subsections we will consider the calculation of the spatial derivative of the local radial functions and the Green function.

\section{B. Plane integration}

First we consider a direct evaluation of the conductance by use of Eq. (10) and calculation of the spacial derivative of the Green function at exactly the plane surfaces left $\left(S_{\mathrm{L}}\right)$ and right $\left(S_{\mathrm{R}}\right)$. Both $S_{\mathrm{L}}$ and $S_{\mathrm{R}}$ are assumed to be in the asymptotic region where the potential is stabilized to the bulk one and the evanescent states have decayed; in practice one has to perform the calculations for several positions of $S_{\mathrm{L}}$ and $S_{\mathrm{R}}$ at finite distances to verify that the results remain unchanged.

The set of atomic cells is volume filling, and the plane surface cutting through them inherits the cellular structure used in the KKR method; thus it is split in two-dimensional tesselating cells. Each one of them is a convex polygon corresponding to the section of the plane that belongs to a convex Voronoi polyhedron ${ }^{46}$ (or just a Wigner-Seitz cell in the monatomic case). In this way, a two-dimensional cellular Voronoi construction is defined in the plane, each cell of which is completely within some three-dimensional cell. Therefore the representation of the Green function in terms of local radial functions can be readily used. In fact, in systems with two-dimensional periodicity, a two-dimensional unit cell consisting of some convex Voronoi polygons $S_{\mu}$ is constructed and the calculations can be confined in those. An example of the construction for bcc (001) surface cells is given in Fig. 3.

Using such a construction, the $\mathbf{k}_{\|}$-resolved conductance is written as

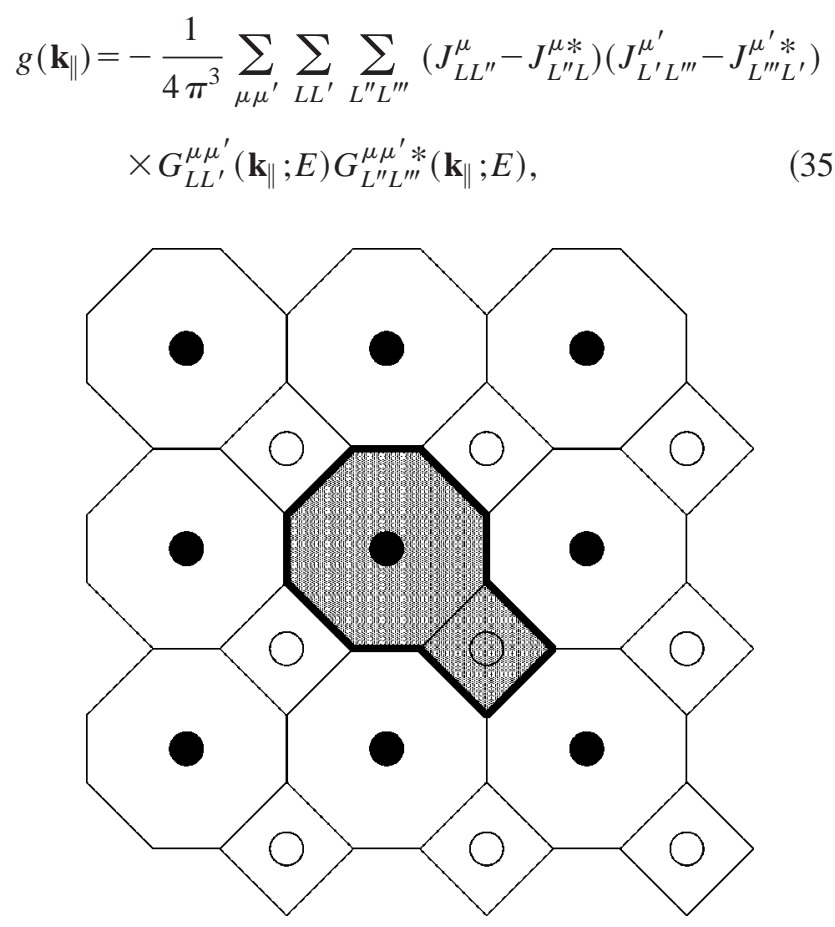

FIG. 3. Two-dimensional geometrical construction for the bcc (001) surface cut. The cutting plane goes through the lattice sites of an atomic layer (full circles), but must include also part of the Wigner-Seitz cell of the next layer (open circles). The shaded area shows the two-dimensional unit cell formed, consisting of two smaller convex Voronoi polygons. 
where we have introduced the KKR current matrix elements $J_{L L^{\prime}}^{\mu}$ (in a similar fashion as has been done in the past ${ }^{7,8}$ ) in a cell as

$$
J_{L L^{\prime}}^{\mu}=\int_{S_{\mu}} d^{2} r R_{L}\left(\mathbf{r} ; E_{F}\right) \partial_{z} R_{L^{\prime}}^{*}\left(\mathbf{r} ; E_{F}\right) .
$$

The summation $\Sigma_{\mu \mu^{\prime}}$ is over the Voronoi polygons of the inequivalent atomic sites of the 2D unit cells in the leads. The calculation of $J_{L L^{\prime}}^{\mu}$ is described in Appendix B. In the case of a finite system, where a two-dimensional Fourier transform is not necessary, the summation is over all atoms in the planes $S_{\mathrm{L}}$ and $S_{\mathrm{R}}$, and the $\mathbf{k}_{\|}$dependence drops.

The Green-function matrix elements $G_{L L^{\prime}}^{\mu \mu^{\prime}}$, containing the information for the propagation from site $\mu^{\prime}$ at a layer within the left lead to a site $\mu$ at a layer in the right lead, are off-diagonal, while for the charge density one needs only the diagonal (on-site) elements. Nevertheless, an efficient $\mathrm{O}(N)$ algorithm exists ${ }^{47}$ for their calculation within the screened KKR formalism, i.e., the time needed for the calculation scales linearly with the distance between the two layers. Thus it is possible to calculate the conductance in junctions of more than 100 monolayers with present-day computers. ${ }^{29,30}$

We note that a formula for the direct calculation of the reflection probability via the Green function can be derived along similar lines [see, for example, Ref. 32, Eq. (76)]. However, this formula uses the on-site Green function, i.e., the term $n=n^{\prime}$ in Eq. (31). Then the irregular solution of the Schrödinger equation is included [denoted as $H_{L}$ in Eq. (31)], which is divergent at the origin. For the evaluation of the current one has to calculate numerically the derivative of this divergent function, and this leads to numerical instability. This is avoided when calculating directly the transmission coefficient, since then we have always $n \neq n^{\prime}$.

\section{Volume integration: Atomic sphere approximation and full cell}

In this section we provide an alternative to the calculation of the surface-integrated current matrix elements of Eqs. (36) and (B4). We prove that the calculation can involve a volume integration over the unit cell, instead of a surface integration; in principle, the results must be equivalent, but this method has advantages when one wishes to use the atomic sphere approximation. Most important is, however, that the $l$ convergence is much better (see Sec. VII).

First we observe that the value of the conductance is indeed independent of the position of the planes $S_{\mathrm{L}}$ and $S_{\mathrm{R}}$. This can be proven using the fact that the conductivity tensor is divergenceless [Eq. (7)]. Say that $\mathbf{r}$ is on $S_{\mathrm{R}}$; if we consider a second plane surface $S_{\mathrm{R}}^{\prime}$ close to $S_{\mathrm{R}}$, we can utilize Gauss's theorem in the volume $V$ enclosed by the two planes to convert a volume integral of Eq. (7) in $V$ into a surface integral over $S_{\mathrm{R}}, S_{\mathrm{R}}^{\prime}$, plus side areas. The construction is analogous to that described in Appendix A, as shown in Fig. 4. The contribution from the side areas vanishes because there we have either totally confining boundary conditions or Born-von Kármán boundary conditions leading to cancella-

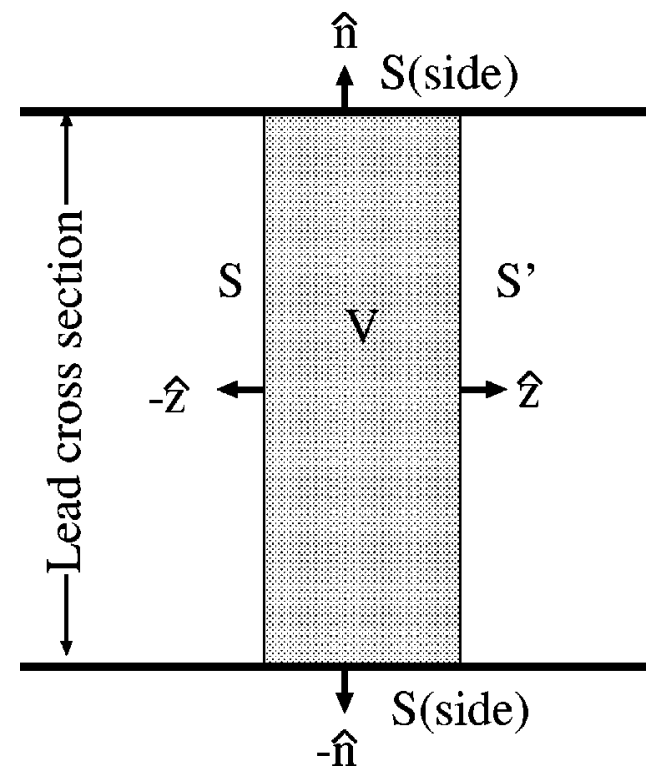

FIG. 4. Construction for the conversion of volume to surface integrals over the lead cross section.

tion from opposite side areas due to the opposite surface unit vector orientation; then we are left with $\int_{S_{\mathrm{R}}} d S \boldsymbol{\sigma}_{i j}\left(\mathbf{r}, \mathbf{r}^{\prime}\right) \cdot \hat{z}$ $=\int_{S_{\mathrm{R}}^{\prime}} d S \boldsymbol{\sigma}_{i j}\left(\mathbf{r}, \mathbf{r}^{\prime}\right) \cdot \hat{z}$. The same applies for $S_{\mathrm{L}}$, where $z^{\prime}$ varies; thus the flux of $\boldsymbol{\sigma}_{i j}\left(\mathbf{r}, \mathbf{r}^{\prime}\right)$, i.e., the conductance, is independent of the exact position of $S_{\mathrm{L}}$ and $S_{\mathrm{R}}$, QED. In fact, following these arguments, we see that $S_{\mathrm{L}}$ and $S_{\mathrm{R}}$ do not even have to be planes; for instance, they can follow the pattern of the Wigner-Seitz or Voronoi cells, as long as they meet the requirement that they satisfy the Born-von Kármán periodic boundary condition in $x$ and $y$. In the case that they are not planar surfaces, one must of course take the flux of the conductivity tensor really along the normal $\hat{\boldsymbol{n}}$ pointing outward at each point of the surfaces, i.e.,

$$
g=\int_{S_{\mathrm{R}}} d S \int_{S_{\mathrm{L}}} d S^{\prime} \hat{\boldsymbol{n}} \cdot \boldsymbol{\sigma}\left(\mathbf{r}, \mathbf{r}^{\prime}\right) \cdot \hat{\boldsymbol{n}}^{\prime}
$$

Since the exact choice of $S_{\mathrm{R}}$ or $S_{\mathrm{L}}$ does not affect the result, one can average over the volume $V$ included between, say, $S_{\mathrm{R}}$ and $S_{\mathrm{R}}^{\prime}$ instead of integrating over $S_{\mathrm{R}}$. In particular, $V$ can be chosen to have a thickness $d$ equivalent to a unit cell in the $z$ direction, so that one has to average over layeradapted unit cells. In this respect, the conductance formula has the same form as Eq. (35), but with an extra prefactor of $1 / d^{2}$ to account for the volume averaging in the two leads $(d$ here is the distance between two consecutive lattice monolayers); the current matrix elements $J_{L L^{\prime \prime}}$, volume-averaged here in the atomic cells, have the form of Eq. (36) but with the integral being three-dimensional over the atomic cell. This can be done both in the atomic sphere approximation (ASA) and in the full-potential (and full-cell) formalism. Details about their calculation are given again in Appendix B.

A word of caution is due here: it is essential that the volume averaging leaves no "holes" in the structure. To be 

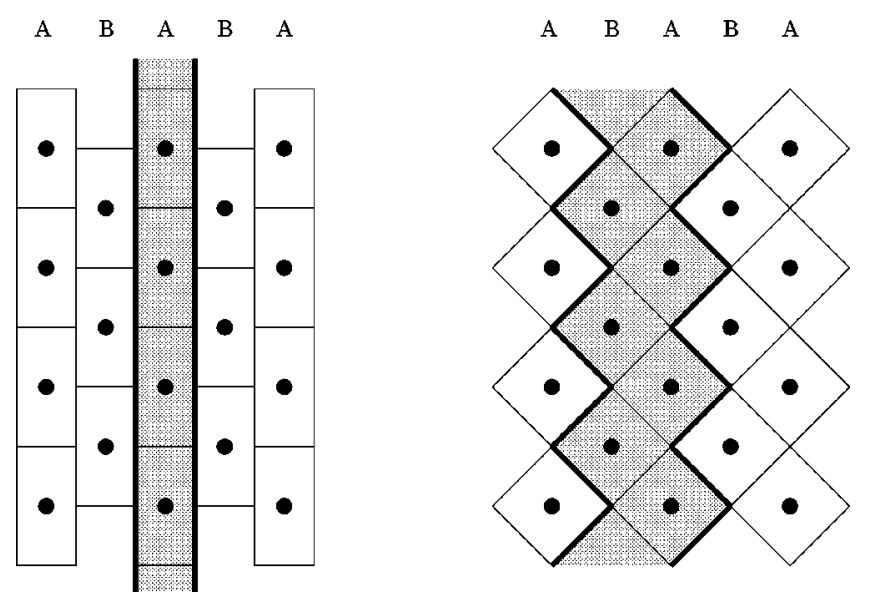

FIG. 5. Two possibilities for cell-averaging of the conductivity tensor flux in an ...ABAB ... stacking sequence. Left, using a cellular division without corrugation of the surfaces, and right, using the Wigner-Seitz construction with corrugation. The latter is preferable in the KKR method.

specific, assume a bcc-like structure. Then, as is evident from Fig. 3, the Voronoi cells of the first layer (open circles) can touch via the "holes" (corresponding to the squares) to the cells of the third layer. In this way, the current can partly bypass one monolayer traveling directly to the next one. If one just takes the current averaged over the Voronoi cells or the ASA spheres of the middle monolayer, one forgets to calculate this part of the current; this is why the full manyatom unit cell must be taken, so that no such holes are left.

If one uses the usual Wigner-Seitz cells (or atomic spheres), Eq. (B5) can induce a small inaccuracy. The reason is that the volume constructed by such cells is in general not included between planar surfaces, as in Fig. 4, but rather between corrugated surfaces, in accordance to the form of the Wigner-Seitz cells. In such a case, the full conductivity tensor $\boldsymbol{\sigma}\left(\mathbf{r}, \mathbf{r}^{\prime}\right)$ (not just the $\boldsymbol{\sigma}_{z z}$ component) and Eq. (37) should be used in principle. To avoid such a more complicated calculation, two ways can be followed, as demonstrated in Fig. 5. First, one can persist in using layer-adapted unit cells (parellelepipeds), which give no corrugation. This has the disadvantage that such cells can be too flat so that the $l$ expansion of the cell-centered KKR Green function and wave function converges poorly. Second, one can average over more than one monolayers; in this example they would be $A$ and $B$ (Fig. 4, right panel) taken on both the right and left leads. Then the corrugated region is a smaller fraction of the total averaging volume, so that the error becomes smaller. Test calculations on this will be given in the Sec. VII for bulk Al.

\section{Current matrix elements and selection rules}

Which method, volume or plane integration, is most convenient and accurate depends on each specific problem; however one can have "rules of thumb" on the difficulty and convergence of each one. In many systems the atomic sphere approximation is used, where the potential around each site is assumed to be spherically symmetric, but still a full mul- tipole expansion of the charge density is taken. This has the advantage of greater simplicity and less computational effort than a full potential and full cell description. In such cases, the plane integration is not applicable, since a plane cut through volume-conserving spheres cannot give an accurate surface area; the volume integration is then the only way. In the case of a full-cell treatment, when one has the correct Wigner-Seitz or Voronoi volume tesselation, one must consider that the plane method has a drawback, namely, that the plane might go through regions only at the edge of certain cells, where the $l_{\max }$ cutoff seriously affects the accuracy of the results; on the other hand the volume integration averages out such inaccuracies.

One has also "selection rules" that make certain $J_{L L^{\prime}}$ elements vanish. This is most easily seen if one uses spherical potentials. To be specific, say that the plane goes through the atomic site at $z=0$; then, in the plane integration one can easily see that the elements $J_{L L^{\prime}}$ are nonvanishing for $l^{\prime}$ $=1,3,5, \ldots$ when $l$ is even and for $l^{\prime}=2,4,6, \ldots$ when $l$ is odd. On the other hand, in the case of volume integration the $J_{L L^{\prime}}$ are nonvanishing only for $l^{\prime}=l, l \pm 1$, i.e., $J_{L L^{\prime}}$ is band diagonal in $l$ and $l^{\prime}$. This can be viewed as an advantage of the volume-averaging method, since it means that, if one describes the electronic structure with orbitals truncated at $l_{\max }$, for the accuracy of $J_{L L^{\prime}}$ one has to consider wave functions only up to $l^{\prime}=l_{\max }+1$. A full-cell treatment adds more nonzero elements, but the main contribution still comes from the ones mentioned.

\section{EXAMPLES}

In recent papers we have applied the method presented here to spin-dependent transport in ferromagnet/ semiconductor systems ${ }^{29,30}$ tunneling through vacuum, ${ }^{48}$ and atomic-sized contacts. ${ }^{45}$ In this section we confine the calculations to the simplest examples, in order to demonstrate the convergence properties of the method.

\section{A. Band counting in bulk conductance}

When the Landauer formula is applied to a perfectly periodic material, e.g., the bulk of a crystal, it gives a finite conductance that physically represents the conductance of a long wire placed between two phase-randomizing electrodes. ${ }^{39}$ Resolved in $\mathbf{k}_{\|}$, the value of the conductance equals the number of right-propagating (or equivalently leftpropagating) states at $E_{F}$ for this $\mathbf{k}_{\|}$. In other words, one has to count the Fermi surface bands for that $\mathbf{k}_{\|}$, which propagate in the direction $k_{z} \perp \mathbf{k}_{\|}$with $v_{z}>0$. This is demonstrated in Fig. 6, where part of the Fermi surface of $\mathrm{Al}$ is presented, in the $k_{x}-k_{z}$ plane, together with the conductance in the $z$ direction as a function of $k_{x}$ (with $k_{y}=0$ ). Actually, some of the bands shown have $v_{z}<0$, but their equivalents with $v_{z}$ $>0$ exist symmetrically for $k_{z}<0$. Clearly the conductance (in units of $e^{2} / h$ ) equals the number of bands at $E_{F}$ for each $k_{x}$, giving a stepwise picture.

Also in Fig. 6 we can compare the results for angular momentum truncation at $l_{\max }=2,3$, and 4 . Increasing $l_{\max }$ results in a more accurate description of the wave function 


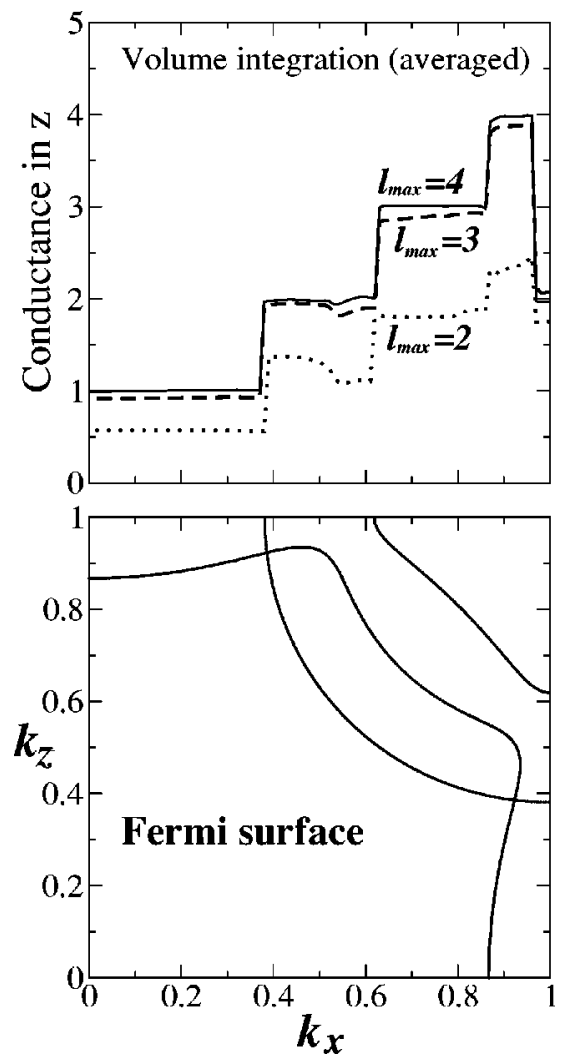

FIG. 6. Fermi surface in the $k_{x}-k_{z}$ plane (bottom) and conductance (in units of $e^{2} / h$ ) as a function of $k_{x}$ (top) for bulk $\mathrm{Al}$ and for $l_{\max }=2,3$, and 4 .

derivative. Basically the slower convergence here arises from the relatively high Fermi energy of $\mathrm{Al}$, and is also present in a free-electron approach for high $E_{F} \cdot{ }^{49}$ As noted in the preceding section, if the wave function is accurate for some $l_{\max }$, for the derivative one has to take $l_{\max }+1$. For most practical purposes, $l_{\max }=3$ is enough, considering also that the calculation time for $l_{\max }=4$ is more than three times the one of $l_{\max }=3$ [due to matrix inversion, the calculation time scales roughly as $\left.\left(l_{\max }+1\right)^{6}\right]$.

When the conductance is analyzed in the various interlayer contributions, these can exhibit fluctuations in $\mathbf{k}_{\|}$, or as a function of the interlayer distance. The origin of these fluctuations is the Wigner-Seitz construction for the unit cell, resulting in corrugation of the surfaces where the current is calculated. Due to the fact that we account only for the $z z$ component of the conductivity tensor, when we have corrugation the matrix elements in Eq. (15) are not integrated correctly and the nondiagonal current matrix elements do not vanish; thus, beating effects of the conductance appear. As the conductance is averaged over more than one monolayers, the corrugation-free region in the middle increases, the relative error due to the corrugation decreases and the steps in the conductance become flat as they should.

Finally, we have made a test calculation for the same system, but employing the in-plane integration, rather than the volume averaging. Here the convergence with $l_{\max }$ is poor for the reasons explained in the preceding section; even for $l_{\max }=4$ the deviations from integer conductance values are large.
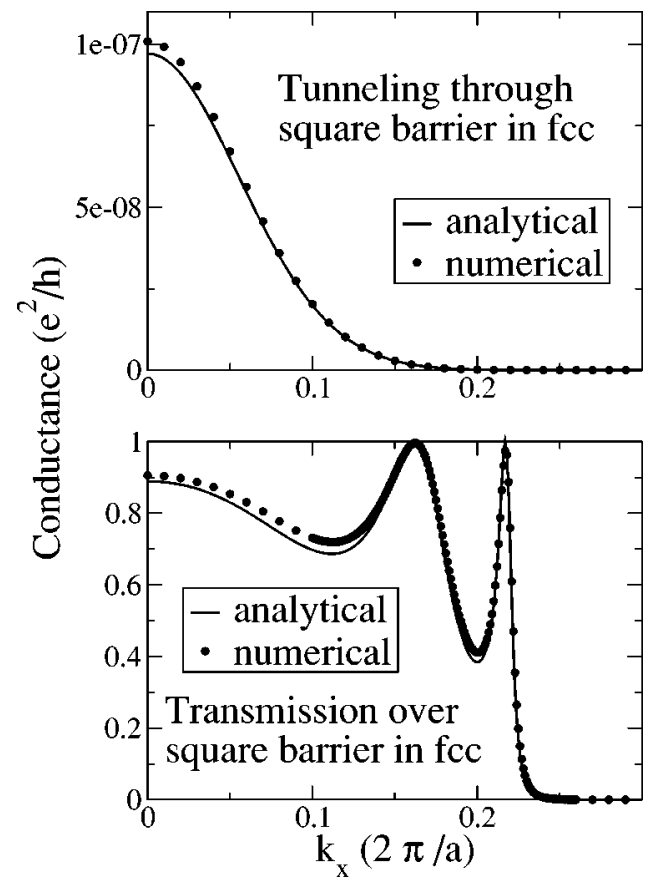

FIG. 7. Transmission through a barrier of constant potential $V_{0}=1 \mathrm{eV}$, sitting on an fcc lattice with lattice constant $a$ $=4.032 \AA(\mathrm{Al})$ and having a thickness $d=6 a$ [twelve (001) monolayers]. Top: Tunneling $\left(E_{F}=0.5 \mathrm{eV}\right)$; bottom: transmission over the barrier $\left(E_{F}=1.5 \mathrm{eV}\right)$, where the transmission resonances are also seen. The full line represents the analytical result for a square barrier.

As a test on the transmission coefficient when scattering is present, we show in Fig. 7 the transmission through a barrier of constant potential $V_{0}=1 \mathrm{eV}$, sitting on an fcc lattice with lattice constant $a=4.032 \AA$ (Al) and having a thickness $d=6 a$ [twelve (001) monolayers]. Two cases are shown: one with $E_{F}=0.5 \mathrm{eV}$ (tunneling) and one with $E_{F}$ $=1.5 \mathrm{eV}$ (transmission over the barrier). The analytical result concerning a square barrier is also shown, with the transmission probability given by

$$
\begin{gathered}
T=\left[1+\left(\frac{k_{z}}{K_{z}}-\frac{K_{z}}{k_{z}}\right)^{2} \frac{\sin ^{2}\left(K_{z} d\right)}{4}\right]^{-1}, E_{F}>V_{0}+\frac{2 m \mathbf{k}_{\|}^{2}}{\hbar^{2}} \\
T=\left[1+\left(\frac{k_{z}}{\kappa}+\frac{\kappa}{k_{z}}\right)^{2} \frac{\sinh ^{2}(\kappa d)}{4}\right]^{-1}, E_{F}<V_{0}+\frac{2 m \mathbf{k}_{\|}^{2}}{\hbar^{2}}
\end{gathered}
$$

with $k_{z}^{2}=2 m E_{F} / \hbar^{2}-\mathbf{k}_{\|}^{2}, \quad K_{z}^{2}=2 m\left(E_{F}-V_{0}\right) / \hbar^{2}-\mathbf{k}_{\|}^{2}$, and $\kappa^{2}=2 m\left(V_{0}-E_{F}\right) / \hbar^{2}+\mathbf{k}_{\|}^{2}$. The agreement is good. Note that a full agreement cannot be expected, since in the lattice model the two interfaces of the barrier are corrugated due to the Wigner-Seitz construction, while the analytical model assumes planar interfaces. However, at small energies or long wavelengths this corrugation does not play a major role.

\section{B. The effect of the nonzero imaginary part of the energy}

Although the conductance should be calculated at a real energy $E$, the Green function in the KKR method is always 


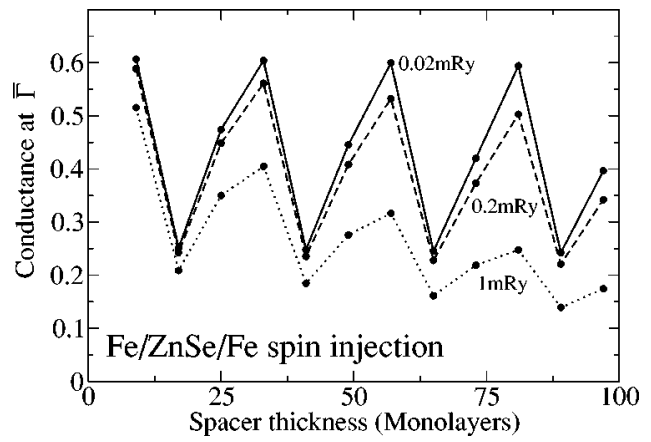

FIG. 8. The effect of the nonzero imaginary part $\epsilon$ of the energy. Conductance (in units of $e^{2} / h$ ) as a function of spacer thickness for spin injection through the conduction band in a $\mathrm{Fe} / \mathrm{ZnSe} / \mathrm{Fe}(001)$ junction. The oscillations are due to multiple reflections. For $\epsilon$ $=1 \mathrm{mRy}$ there is strong artificial damping, while $\epsilon=0.02 \mathrm{mRy}$ is adequate even for the thickness of 97 monolayers.

calculated at a complex energy $E+i \epsilon$, and the real $E$ is approximated by taking very small, but nonzero, $\epsilon$. This can have an artificial damping effect to the conductance, since waves within a small energy range around $E$ are effectively superimposed and finally the phase is randomized, especially if the leads are seperated by a large distance. In Fig. 8 we show an example of how small $\epsilon$ should be in a realistic calculation. The system here consists of two Fe leads with parallel magnetic moment seperated by a ZnSe spacer. Electrons are injected from the first lead into the $\mathrm{ZnSe}$ conduction band, and are detected by the second Fe lead. The spacer thickness is varied from 9 to 97 monolayers, and due to the multiple reflections at the two interfaces transmission resonances appear at certain thicknesses. For more information we refer to Ref. 30. The conductance of the majority electrons for $\mathbf{k}_{\|}=0$, i.e., at the $\bar{\Gamma}$ point, is presented in Fig. 8 for a choice of $\epsilon=0.02 \mathrm{mRy}, 0.2 \mathrm{mRy}$, and $1 \mathrm{mRy}$. For $\epsilon$ $=1 \mathrm{mRy}$ there is strong artificial damping, while $\epsilon$ $=0.02 \mathrm{mRy}$ is adequate even for the large thickness of 97 monolayers. Note that this damping cannot model an effect of temperature, because when we depart from the real axis, the spectral density of a state transforms from a $\delta$ function to a Lorenzian distribution and not to the derivative of the Fermi function. Due to the long tails of the Lorenzian the damping is much stronger than for a Fermi distribution of the same half-width.

\section{SUMMARY AND CONCLUSIONS}

We have presented a formalism for the calculation of ballistic conductance in solids, based on the KKR Greenfunction method for the ground-state electronic structure combined with the Landauer-Büttiker approach. It makes use of the result of Baranger and Stone ${ }^{32}$ connecting the derivative of the one-electron Green function to the conductance. For the foundation of the formalism, we have discussed the relation of the $S$ matrix between Bloch in and out states to the conductance. We have given an expression connecting the $S$ matrix to the Green function of the system, generaliz- ing the theory of Baranger and Stone to include the realistic band structure of the leads.

The convergence of the method with angular momentum cutoff $\left(l_{\max }\right)$ was studied and found to be comparable to that of KKR. It can be applied to systems with two-dimensional periodicity as well as nanowires. Our results show that the volume integration and averaging of the current matrix elements, applicable both in ASA and full-cell or full-potential approaches, gives well-converged results for the calculation of ballistic transport. Owing to the linear scaling of the calculational effort with the number of layers of the screened KKR formalism $[\mathrm{O}(N)$ scaling], our method is suitable for large systems.

\section{ACKNOWLEDGMENTS}

The authors are grateful to Professor N. Stefanou for helpful and motivating discussions. Moreover, financial support from the RT Network of Computational Magnetoelectronics (Contract No. RTN1-1999-00145) of the European Commission is gratefully acknowledged.

\section{APPENDIX A: CURRENT MATRIX ORTHOGONALITY RELATIONS}

Let $\Psi_{\mathbf{k} a}$ and $\Psi_{\mathbf{k}^{\prime} a^{\prime}}$ be two Bloch wave functions of the same Hamiltonian at the same energy. Then we shall prove that the following relation holds:

$$
\begin{aligned}
\int_{S} d S\left(\mathbf{W}_{\mathbf{k} a ; \mathbf{k}^{\prime} a^{\prime}}\right)_{z} & :=\int_{S} d S\left(\Psi_{\mathbf{k} a}^{*} \overleftrightarrow{\nabla}_{z} \Psi_{\mathbf{k}^{\prime} a^{\prime}}\right) \\
& =i \frac{2 m}{\hbar}\left(v_{\mathbf{k} a}\right)_{z} \delta_{\mathbf{k k}^{\prime}} \delta_{a a^{\prime}},
\end{aligned}
$$

where $S$ is an (infinite) cross-sectional area in $x$ and $y$ directions.

The proof has as follows: First we note that, as a consequence of the single-particle Schrödinger equation for a real potential,

$$
\begin{aligned}
\boldsymbol{\nabla} \mathbf{W}_{\mathbf{k} a ; \mathbf{k}^{\prime} a^{\prime}} & =\boldsymbol{\nabla}\left(\Psi_{\mathbf{k} a}^{*} \overleftrightarrow{\nabla} \Psi_{\mathbf{k}^{\prime} a^{\prime}}\right) \\
& =-\frac{2 m}{\hbar}\left(E_{\mathbf{k}^{\prime} a^{\prime}}-E_{\mathbf{k} a}\right) \Psi_{\mathbf{k} a}^{*} \Psi_{\mathbf{k}^{\prime} a^{\prime}} \\
& =0 \text { for } E_{\mathbf{k}^{\prime} a^{\prime}}=E_{\mathbf{k} a},
\end{aligned}
$$

where the band indices $a$ and $b$ are used explicitly. This is just an expression for current conservation of Hamiltonian eigenstates. Then, for each volume $V$ enclosed by a geometrical surface $S$, Gauss's theorem gives

$$
\oint_{S} d S \hat{\boldsymbol{n}} \cdot \mathbf{W}_{\mathbf{k} a ; \mathbf{k}^{\prime} a^{\prime}}=\int_{V} d^{3} r \quad \nabla \mathbf{W}_{\mathbf{k} a ; \mathbf{k}^{\prime} a^{\prime}}=0,
$$

where $\hat{\boldsymbol{n}}$ is a unit vector at the surface $S$ pointing outward. In particular, $V$ can be chosen as a prismatic normal cross section of the lead, extending from $z$ to $z+d$. Then its surface $S$ can be decomposed in two plane cross sections $S_{1}$ at $z$ and $S_{2}$ at $z+d$, as the bases of the prism, plus side areas $S_{\text {side }}$ at the 
lead surface, as shown in Fig. 4. At these side areas we either have confining boundary conditions, i.e., $\left.\Psi_{\mathbf{k} a}\right|_{\text {side }}=0$, whence $\left.\mathbf{W}_{\mathbf{k} a ; \mathbf{k}^{\prime} a^{\prime}}\right|_{\text {side }}=0$ or Born-von Karman periodic boundary conditions, whence for each prism side there is the opposite one with the same value of $\left.\Psi_{\mathbf{k} a}\right|_{\text {side }}$ and $\left.\mathbf{W}_{\mathbf{k} a ; \mathbf{k}^{\prime} a^{\prime}}\right|_{\text {side }}$ but with opposite orientation unit vector $\hat{\boldsymbol{n}}$; then the sum of their contributions to the surface integral will be again zero. In this way, we are left with the two bases of the prism; they have opposite unit vector orientations, thus

$$
\int_{S_{1}} d S_{1}(-\hat{z}) \cdot \mathbf{W}_{\mathbf{k} a ; \mathbf{k}^{\prime} a^{\prime}}+\int_{S_{2}} d S_{2} \hat{z} \cdot \mathbf{W}_{\mathbf{k} a ; \mathbf{k}^{\prime} a^{\prime}}=0
$$

or

$$
\int_{S_{1}} d S_{1}\left(\mathbf{W}_{\mathbf{k} a ; \mathbf{k}^{\prime} a^{\prime}}\right)_{z}=\int_{S_{2}} d S_{2}\left(\mathbf{W}_{\mathbf{k} a ; \mathbf{k}^{\prime} a^{\prime}}\right)_{z},
$$

i.e., $\int_{S} d S\left(\mathbf{W}_{\mathbf{k} a ; \mathbf{k}^{\prime} a^{\prime}}\right)_{z}$ is independent of the position $z$ of the cross section $S$. This means that one can average it over the whole Born-von Kármán supercell (in $z$ ) of length $L$ and volume $V_{\mathrm{BvK}}=S L$ :

$$
\begin{aligned}
\int_{S} d S\left(\mathbf{W}_{\mathbf{k} a ; \mathbf{k}^{\prime} a^{\prime}}\right)_{z} & =\frac{1}{L} \int_{0}^{L} d z \int d S\left(\mathbf{W}_{\mathbf{k} a ; \mathbf{k}^{\prime} a^{\prime}}\right)_{z}(x, y, z) \\
& =\frac{1}{L} \int_{V_{\mathrm{BvK}}} d^{3} r\left(\mathbf{W}_{\mathbf{k} a ; \mathbf{k}^{\prime} a^{\prime}}\right)_{z}(x, y, z) .
\end{aligned}
$$

We now convert the volume integral in a sum over unit-cell integrals $\Sigma_{i} \int_{V_{0}} d^{3} r$ and employ the Bloch property

$$
\mathbf{W}_{\mathbf{k} a ; \mathbf{k}^{\prime} a^{\prime}}\left(\mathbf{R}_{i}+\mathbf{r}\right)=e^{i\left(\mathbf{k}^{\prime}-\mathbf{k}\right) \cdot \mathbf{R}_{i} \mathbf{W}_{\mathbf{k} a ; \mathbf{k}^{\prime} a^{\prime}}(\mathbf{r})}
$$

to get

$$
\begin{aligned}
\int_{S} d S\left(\mathbf{W}_{\mathbf{k} a ; \mathbf{k}^{\prime} a^{\prime}}\right)_{z}(x, y, z) & =\frac{1}{L} \sum_{i} \int_{V_{0}} d^{3} r\left(\mathbf{W}_{\mathbf{k} a ; \mathbf{k}^{\prime} a^{\prime}}\right)_{z}\left(\mathbf{R}_{i}+\mathbf{r}\right) \\
& =\frac{N}{L} \delta_{\mathbf{k k}^{\prime}} \int_{V_{0}} d^{3} r\left(\mathbf{W}_{\mathbf{k} a ; \mathbf{k}^{\prime} a^{\prime}}\right)_{z}(\mathbf{r}),
\end{aligned}
$$

where $N$ is the total number of lattice sites in $V_{\mathrm{BvK}}$, and

$$
\sum_{i} e^{i\left(\mathbf{k}^{\prime}-\mathbf{k}\right) \cdot \mathbf{R}_{i}}=N \delta_{\mathbf{k k}^{\prime}}
$$

has been used; $V_{0}$ is the unit-cell volume. Note that $N V_{0}$ $=V_{\mathrm{BvK}}=S L$, thus $N / L=S / V_{0}$, and we get

$$
\int_{S} d S\left(\mathbf{W}_{\mathbf{k} a ; \mathbf{k}^{\prime} a^{\prime}}\right)_{z}(x, y, z)=\frac{S}{V_{0}} \delta_{\mathbf{k k}^{\prime}} \int_{V_{0}} d^{3} r\left(\mathbf{W}_{\mathbf{k} a ; \mathbf{k}^{\prime} a^{\prime}}\right)_{z}(\mathbf{r}) .
$$

For $\mathbf{k} \neq \mathbf{k}^{\prime}$ this gives zero, while for $\mathbf{k}=\mathbf{k}^{\prime}$ and $a=a^{\prime}$ we have

$$
\begin{aligned}
\int_{V_{0}} d^{3} r\left(\mathbf{W}_{\mathbf{k} a ; \mathbf{k} a}\right)_{z}(\mathbf{r}) \\
\quad=\frac{i m}{\hbar} \int_{V_{0}} d^{3} r\left(\Psi_{\mathbf{k} a}^{*} \frac{\hbar}{i m} \boldsymbol{\nabla} \Psi_{\mathbf{k} a}-\Psi_{\mathbf{k} a} \frac{\hbar}{i m} \nabla \Psi_{\mathbf{k} a}^{*}\right)_{z} \\
=i \frac{2 m}{\hbar}\left(\mathbf{v}_{\mathbf{k} a}\right)_{z} .
\end{aligned}
$$

Equations (A12) and (A11) verify Eq. (A1) except in the case of band crossing, when $\mathbf{k}=\mathbf{k}^{\prime}$ but $a \neq a^{\prime}$. In this case we may use the identity connecting the momentum operator $\mathbf{p}_{\text {op }}$ to the Hamiltonian $H$ and the position operator $\mathbf{r}_{\mathrm{op}}$ :

$$
\mathbf{p}_{\mathrm{op}}=\frac{i m}{\hbar}\left[H, \mathbf{r}_{\mathrm{op}}\right]
$$

For the evaluation of Eq. (A6) we need the matrix element of $\mathbf{p}_{\text {op }}:=(\hbar / i) \boldsymbol{\nabla}$. Using Eq. (A13) we get

$$
\begin{aligned}
& \frac{\hbar}{i m} \int_{V_{\mathrm{BvK}}} d^{3} r \Psi_{\mathbf{k} a}^{*} \mathbf{p}_{\mathrm{op}} \Psi_{\mathbf{k}^{\prime} a^{\prime}} \\
& \quad=\left(E_{\mathbf{k} a}-E_{\mathbf{k}^{\prime} a^{\prime}}\right) \int_{V_{\mathrm{BvK}}} d^{3} r \Psi_{\mathbf{k} a}^{*} \mathbf{r} \Psi_{\mathbf{k}^{\prime} a^{\prime}} .
\end{aligned}
$$

In the case of band crossing, $E_{\mathbf{k} a}-E_{\mathbf{k}^{\prime} a^{\prime}}=0$, but this does not mean that the whole expression vanishes, since the integral might diverge. It can be calculated in a standard way by utilizing Bloch's theorem and reducing it to the unit cell. We have

$$
\begin{aligned}
& \int_{V_{\mathrm{BvK}}} d^{3} r \Psi_{\mathbf{k} a}^{*}(\mathbf{r}) \mathbf{r} \Psi_{\mathbf{k}^{\prime} a^{\prime}}(\mathbf{r}) \\
& \quad=\sum_{i} \int_{V_{0}} d^{3} r \Psi_{\mathbf{k} a}^{*}\left(\mathbf{R}_{i}+\mathbf{r}\right)\left(\mathbf{R}_{i}+\mathbf{r}\right) \Psi_{\mathbf{k}^{\prime} a^{\prime}}\left(\mathbf{R}_{i}+\mathbf{r}\right)
\end{aligned}
$$

Using the Bloch properties of $\Psi_{\mathbf{k} a}$ and $\Psi_{\mathbf{k}^{\prime} a^{\prime}}$ and the relations

$$
\sum_{i} e^{i\left(\mathbf{k}^{\prime}-\mathbf{k}\right) \cdot \mathbf{R}_{i}}=\frac{(2 \pi)^{3}}{V_{0}} \delta\left(\mathbf{k}^{\prime}-\mathbf{k}\right)
$$

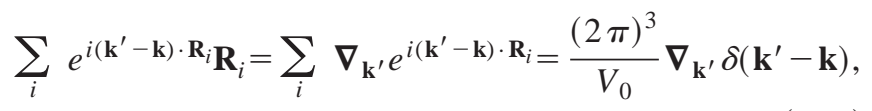

we obtain after some manipulations 


$$
\begin{aligned}
\frac{\hbar}{i m} \int_{V_{\mathrm{BvK}}} d^{3} r \Psi_{\mathbf{k} a}^{*} \mathbf{p}_{\mathrm{op}} \Psi_{\mathbf{k}^{\prime} a^{\prime}} \\
=\frac{(2 \pi)^{3}}{V_{0}} \delta\left(\mathbf{k}^{\prime}-\mathbf{k}\right)\left\{\left(E_{\mathbf{k} a}-E_{\mathbf{k}^{\prime} a^{\prime}}\right) \int_{V_{0}} d^{3} r \Psi_{\mathbf{k} a}^{*} \mathbf{r} \Psi_{\mathbf{k}^{\prime} a^{\prime}}\right. \\
\left.-\hbar \mathbf{v}_{\mathbf{k} a} \delta_{a a^{\prime}}-\left(E_{\mathbf{k} a}-E_{\mathbf{k}^{\prime} a^{\prime}}\right) \nabla_{\mathbf{k}} \int_{V_{0}} d^{3} r \Psi_{\mathbf{k} a}^{*} \Psi_{\mathbf{k}^{\prime} a^{\prime}}\right\} .
\end{aligned}
$$

In all terms, $\mathbf{k}=\mathbf{k}^{\prime}$ can be directly substituted due to the $\delta$ function, except in the last one, where one must first perform the integration and the derivation. In the second term, the orthogonality relation $\int_{V_{0}} d^{3} r \Psi_{\mathbf{k}^{\prime} a}^{*} \Psi_{\mathbf{k}^{\prime} a^{\prime}}=\delta_{a a^{\prime}}$ after the substitution $\mathbf{k}=\mathbf{k}^{\prime}$ has been used. From this expression we immediately see that in the case of band crossing, i.e., $\mathbf{k}$ $=\mathbf{k}^{\prime}, E_{\mathbf{k} a}=E_{\mathbf{k}^{\prime} a^{\prime}}$, but $a \neq a^{\prime}$, the expression vanishes, so the proof is complete. In passing we note that, if $a=a^{\prime}$, the expression gives the group velocity as expected.

\section{APPENDIX B: CURRENT MATRIX ELEMENTS IN THE KKR METHOD}

In the plane-integration formalism the KKR current matrix elements read

$$
J_{L L^{\prime}}^{i}=\int_{S_{i}} d^{2} r R_{L}\left(\mathbf{r} ; E_{F}\right) \partial_{z} R_{L^{\prime}}^{*}\left(\mathbf{r} ; E_{F}\right),
$$

where $S_{i}$ is the surface cut of the atomic cell $i$ with the plane passing through it. In the full-potential KKR formalism, the wave functions are expanded in terms of real spherical harmonics as

$$
R_{L}(\mathbf{r})=\sum_{L_{1}} \frac{1}{r} R_{L_{1} L}(r) Y_{L_{1}}(\theta, \phi) .
$$

The real spherical harmonics are of the form

$$
Y_{L}(\theta, \phi)=\alpha_{L} P_{l}^{|m|}(\cos \theta) \operatorname{trg}(m \phi),
$$

where

$$
\begin{gathered}
\alpha_{L}=\sqrt{\frac{2 l+1}{2 \pi} \frac{(l-|m|) !}{(l+|m|) !}}, \quad m \neq 0 \\
\alpha_{L}=\sqrt{\frac{2 l+1}{4 \pi}}, \quad m=0
\end{gathered}
$$

$P_{l}^{|m|}(\cos \theta)$ are the Legendre functions, and $\operatorname{trg}(m \phi)$ $=\cos m \phi$ if $m \geqslant 0$ or $\sin |m| \phi$ if $m<0$. Thus one has to decompose $\partial_{z}$ into $\partial_{r}, \partial_{\theta}$, and $\partial_{\phi}$. The first affects only the radial part $R_{L_{1} L}(r)$ and is calculated numerically; the other two affect only $Y_{L_{1}}(\theta, \phi)$ and are calculated analytically. After some algebra one arrives at the result

$$
\begin{aligned}
J_{L L^{\prime}}= & \int_{r_{\min }}^{r_{\max }} d r \frac{1}{r^{2}} \sum_{L_{2}} \alpha_{L_{2}}\left\{\left[r \partial_{r} R_{L_{2} L^{\prime}}^{*}(r)-\left(l_{2}+1\right) R_{L_{2} L^{\prime}}^{*}(r)\right]\right. \\
& \left.\times u P_{l_{2}}^{\left|m_{2}\right|}(\cos \theta)+\left(l_{2}+\left|m_{2}\right|\right) P_{l_{2}-1}^{\left|m_{2}\right|}(\cos \theta) R_{L_{2} L^{\prime}}^{*}(r)\right\} \\
& \times \sum_{L_{1}} \alpha_{L_{1}} P_{l_{1}}^{\left|m_{1}\right|}(\cos \theta) R_{L_{1} L}(r) \\
& \times \sum_{j} \int_{\phi_{\text {entry }}^{j}}^{\phi_{\text {exit }}^{j}} \operatorname{trg}\left(m_{1} \phi\right) \operatorname{trg}\left(m_{2} \phi\right) d \phi .
\end{aligned}
$$

Here, $r_{\min }$ and $r_{\max }$ are the radii of the inscribed and circumscribed circles, respectively, of the convex polygon, on which the $\phi$ integration is performed, with center the $z$ projection of the atomic site on the plane; $\phi_{\text {entry }}^{j}$ and $\phi_{\text {exit }}^{j}$ are respectively angles of entry into and exit from the convex polygon as the $\phi$ integration is performed.

In the volume-averaging formalism, the KKR current matrix elements have the form

$$
J_{L L^{\prime}}^{i}=\int_{\text {cell }} d^{3} r R_{L}^{i}(\mathbf{r}) \partial_{z} R_{L^{\prime}}^{i *}(\mathbf{r})
$$

These can be computed within the full-cell or ASA formalism; here we shall present both results.

In the full-cell approach, the potential is truncated at the boundary of the Voronoi atomic cell. This is achieved by introducing the characteristic, or "shape," functions $\Theta(\mathbf{r})$, being equal to unity in the cell and vanishing outside. ${ }^{50}$ Their expansion in spherical harmonics,

$$
\Theta(\mathbf{r})=\sum_{L} \Theta_{L}(r) Y_{L}(\theta, \phi)
$$

is used in the calculation of the current matrix elements. After some manipulations we obtain

$$
J_{L L^{\prime}}=\int_{\mathrm{WS}} d^{3} r \Theta(\mathbf{r}) R_{L}(\mathbf{r}) \partial_{z} R_{L^{\prime}}^{*}(\mathbf{r})
$$

$$
\begin{aligned}
& =\sum_{L_{1}} \sum_{L_{2}} \sum_{L_{3}}\left[\int d r \left(R_{L_{1} L}(r) \partial_{r} R_{L_{2} L^{\prime}}^{*}(r)\right.\right. \\
& \left.-\frac{l_{2}+1}{r} R_{L_{1} L}(r) R_{L_{2} L^{\prime}}^{*}(r)\right) \Theta_{L_{3}}(r) \frac{1}{\alpha_{1,0}} \\
& \quad \times \sum_{L_{4}} C_{L_{1} L_{2} L_{4}} C_{1,0 L_{3} L_{4}} \\
& +\int d r \frac{1}{r} R_{L_{1} L}(r) R_{L_{2} L^{\prime}}^{*}(r) \Theta_{L_{3}}(r) \\
& \left.\quad \times \frac{\alpha_{l_{2} m_{2}}}{\alpha_{l_{2}-1, m_{2}}}\left(l_{2}+\left|m_{2}\right|\right) C_{L_{1} L_{3} l_{2}-1, m_{2}}\right]
\end{aligned}
$$


where $C_{L_{1} L_{2} L_{3}}=\int d \Omega Y_{L_{1}}(\hat{\boldsymbol{r}}) Y_{L_{2}}(\hat{\boldsymbol{r}}) Y_{L_{3}}(\hat{\boldsymbol{r}})$ are the Gaunt coefficients and the identity $\int d \Omega Y_{L_{0}} Y_{L_{1}} Y_{L_{2}} Y_{L_{3}}$ $=\Sigma_{L_{4}} C_{L_{1} L_{2} L_{4}} C_{L_{0} L_{3} L_{4}}$ has been used.

The ASA result is simpler since it does not involve the shape functions. The local orbitals have only a spherical part,

$$
R_{L}(\mathbf{r})=\frac{1}{r} R_{l}(r) Y_{L}(\Omega),
$$

whence the current matrix elements become

$$
\begin{aligned}
J_{L L^{\prime}}= & \frac{1}{\alpha_{1,0}} C_{L L^{\prime} ; 1,0} \int d r R_{l}(r) \partial_{r} R_{l^{\prime}}^{*}(r) \\
& -\frac{l^{\prime}+1}{\alpha_{1,0}} C_{L L^{\prime} ; 1,0} \int d r \frac{1}{r} R_{l}(r) R_{l^{\prime}}^{*}(r) \\
& +\frac{\left(l^{\prime}+\left|m^{\prime}\right|\right) \alpha_{l^{\prime} m^{\prime}}}{\alpha_{l^{\prime}-1, m^{\prime}}} \delta_{l l^{\prime}-1} \delta_{m m^{\prime}} \int d r \frac{1}{r} R_{l}(r) R_{l^{\prime}}^{*}(r) .
\end{aligned}
$$

It is implied that the integrals are within the atomic sphere.
${ }^{1}$ R. Kubo, J. Phys. Soc. Jpn. 12, 570 (1957)

${ }^{2}$ P. Weinberger, Phys. Rep. 377, 281 (2003).

${ }^{3}$ I. Mertig, R. Zeller, and P.H. Dederichs, Phys. Rev. B 47, 16178 (1993); T. Vojta, I. Mertig, and R. Zeller, ibid. 46, 15761 (1992).

${ }^{4}$ I. Mertig, Rep. Prog. Phys. 62, 237 (1999).

${ }^{5}$ P. Zahn, J. Binder, I. Mertig, R. Zeller, and P.H. Dederichs, Phys. Rev. Lett. 80, 4309 (1998).

${ }^{6}$ D.A. Greenwood, Proc. Phys. Soc. London 71, 585 (1958).

${ }^{7}$ W.H. Butler, Phys. Rev. B 31, 3260 (1995).

${ }^{8}$ J. Banhart, H. Ebert, P. Weinberger, and J. Voitländer, Phys. Rev. B 50, 2104 (1994).

${ }^{9}$ P. Weinberger, P.M. Levy, J. Banhart, L. Szunyogh, and P. Újfalussy, J. Phys.: Condens. Matter 8, 7677 (1996).

${ }^{10}$ I. Turek, J. Kudrnovský, V. Drchal, L. Szunyogh, and P. Weinberger, Phys. Rev. B 65, 125101 (2002).

${ }^{11}$ J.B. Pendry, Low-Energy Electron Diffraction (Academic Press, New York, 1974).

${ }^{12}$ A. Modinos, Field, Thermionic, and Secondary Electron Emission Spectroscopy (Plenum Press, New York, 1984).

${ }^{13}$ J.M. MacLaren, X.-G. Zhang, W.H. Butler, and X. Wang, Phys. Rev. B 59, 5470 (1999).

${ }^{14}$ M.D. Stiles and D.R. Hamann, Phys. Rev. B 38, 2021 (1988).

${ }^{15}$ J. Mathon, A. Umerski, and M.A. Villeret, Phys. Rev. B 55, 14378 (1997).

${ }^{16}$ E.Y. Tsymbal and D.G. Pettifor, Phys. Rev. B 64, 212401 (2001); D. Kechrakos, E.Y. Tsymbal, and D.G. Pettifor, J. Magn. Magn. Mater. 242-245, 457 (2002).

${ }^{17}$ P.S. Krstić, X.-G. Zhang, and W.H. Butler, Phys. Rev. B 66, 205319 (2002).

${ }^{18}$ D. Wortmann, H. Ishida, and S. Blügel, Phys. Rev. B 65, 165103 (2002).

${ }^{19}$ D. Wortmann, H. Ishida, and S. Blügel, Phys. Rev. B 66, 075113 (2002)

${ }^{20}$ R. Landauer, IBM J. Res. Dev. 1, 223 (1957); 32, 306 (1988).

${ }^{21}$ M. Büttiker, Phys. Rev. Lett. 57, 1761 (1986); IBM J. Res. Dev. 32, 317 (1988).

${ }^{22}$ H.E. Camblong, S. Zhang, and P.M. Levy, Phys. Rev. B 47, 4735 (1993).

${ }^{23}$ X.-G. Zhang and W.H. Butler, Phys. Rev. B 51, 10085 (1995).

${ }^{24}$ H. Itoh, J. Inoue, and S. Maekawa, Phys. Rev. B 51, 342 (1995).

${ }^{25}$ K.M. Schep, P.J. Kelly, and G.E.W. Bauer, Phys. Rev. B 57, 8907 (1998).
${ }^{26}$ C. Caroli, R. Combescot, D. Lederer, P. Nizieres, and D. SaintJames, J. Phys. C 4, 2598 (1971).

${ }^{27}$ S. Zhang and P.M. Levy, Eur. Phys. J. B 10, 599 (1999).

${ }^{28}$ P. Weinberger, V. Drchal, J. Kudrnovský, I. Turek, H. Herper, L. Szunyogh, and C. Sommers, Philos. Mag. B 82, 1027 (2002).

${ }^{29}$ O. Wunnicke, Ph. Mavropoulos, R. Zeller, P.H. Dederichs, and D. Grundler, Phys. Rev. B 65, 241306(R) (2002); M. Freyss, N. Papanikolaou, V. Bellini, R. Zeller, P.H. Dederichs, and I. Turek, J. Magn. Magn. Mater. 240, 117 (2002); O. Wunnicke, Ph. Mavropoulos, and P.H. Dederichs, Phase Transitions 76, 511 (2003); O. Wunnicke, Ph. Mavropoulos, and P.H. Dederichs, J. Supercond. 16, 171 (2003).

${ }^{30} \mathrm{Ph}$. Mavropoulos, O. Wunnicke, and P.H. Dederichs, Phys. Rev. B 66, 024416 (2002).

${ }^{31}$ M. Zwierzycki, K. Xia, P.J. Kelly, G.E.W. Bauer, and I. Turek, Phys. Rev. B 67, 092401 (2003).

${ }^{32}$ H.U. Baranger and A.D. Stone, Phys. Rev. B 40, 8169 (1989).

${ }^{33}$ H. Ohnishi, Y. Kondo, and K. Takayanagi, Nature (London) 395, 780 (1998).

${ }^{34}$ K. Wildberger, R. Zeller, and P.H. Dederichs, Phys. Rev. B 55, 10074 (1997); N. Papanikolaou, R. Zeller, and P.H. Dederichs, J. Phys.: Condens. Matter 14, 2799 (2002).

${ }^{35}$ A.D. Stone and A. Szafer, IBM J. Res. Dev. 32, 384 (1988).

${ }^{36}$ D.S. Fisher and P.A. Lee, Phys. Rev. B 23, 6851 (1981).

${ }^{37}$ J. Rammer, Rev. Mod. Phys. 63, 781 (1991).

${ }^{38}$ T.N. Todorov, G.A.D. Briggs, and A.P. Sutton, J. Phys.: Condens. Matter 5, 2389 (1993).

${ }^{39}$ Y. Imry and R. Landauer, Rev. Mod. Phys. 71, S306 (1999).

${ }^{40}$ This can be best understood in a simple one-dimensional model of a free electron of wave number $k_{\text {in }}=\sqrt{E}$ encountering an infinitely long step barrier of height $V_{0}$, where the wave number is $k_{\text {out }}=\sqrt{E-V_{0}}$. The transmission amplitude is $t=2 k_{\text {in }} /\left(k_{\text {out }}\right.$ $\left.+k_{\text {in }}\right)$, while the transmission probability is $T=4 k_{\text {out }} k_{\text {in }} /\left(k_{\text {out }}\right.$ $\left.+k_{\text {in }}\right)^{2}=|t|^{2} k_{\text {in }} / k_{\text {out }}$.

${ }^{41}$ Use of these orthogonality relations has been made in many cases in the past; see, for example, P.M. Marcus and D.W. Jepsen, Phys. Rev. Lett. 20, 925 (1968).

${ }^{42}$ L. I. Schiff, Quantum Mechanics, 3rd ed. (McGraw-Hill, New York, 1968); R.G. Newton, Scattering Theory of Waves and Particles, 2nd ed. (Springer, New York, 1982); A. Bohm, Quantum Mechanics: Foundations and Applications, 2nd ed. (Springer, New York, 1986).

${ }^{43}$ J. Opitz, P. Zahn, and I. Mertig, Phys. Rev. B 66, 245417 (2002). 
${ }^{44}$ N. Papanikolaou, J. Opitz, P. Zahn, and I. Mertig, Phys. Rev. B 66, 165441 (2002).

${ }^{45}$ A. Bagrets, N. Papanikolaou, and I. Mertig, cond-mat/0303480 (2003) (unpublished).

${ }^{46}$ We remind the reader that Voronoi polyhedra are volume-filling convex polyhedra around the atomic sites that correspond to a Wigner-Seitz construction, but for arbitrary (not necessarily periodic) positions of the atomic sites; moreover, one can choose a certain "weight" for each atom type in order to give it more or less volume fraction.

${ }^{47}$ E. Godfrin, J. Phys.: Condens. Matter 3, 7843 (1991); V. Bellini, Ph.D. thesis, Forschungszentrum, Jülich (2000).

${ }^{48}$ O. Wunnicke, N. Papanikolaou, R. Zeller, P.H. Dederichs, V. Drchal, and J. Kudrnovský, Phys. Rev. B 65, 064425 (2002).
${ }^{49}$ The connection between $E_{F}$ and $l_{\max }$ convergence can be understood if one considers the $l$ expansion of the free electron Green's function, which is a sum of products of Bessel and Hankel functions with spherical harmonics. The argument of the Bessel and Hankel functions is $\sqrt{E} r$. So, smaller $\sqrt{E_{F}}$ or smaller $r$ means faster $l$ convergence. Thus, as a rule of thumb, $l$ convergence is better for smaller energies and smaller atomic spheres. The energy zero is identified with the average potential in the interstitial region, i.e., in the space outside the touching muffin-tin spheres. Usually, this is near the bottom of the band.

${ }^{50}$ N. Stefanou, H. Akai, and R. Zeller, Comput. Phys. Commun. 60, 231 (1990); N. Stefanou and R. Zeller, J. Phys.: Condens. Matter 3, 7599 (1991). 